\title{
EIGENVALUES FOR THE ROBIN LAPLACIAN IN DOMAINS WITH VARIABLE CURVATURE
}

\author{
BERNARD HELFFER AND AYMAN KACHMAR
}

\begin{abstract}
We determine accurate asymptotics for the low-lying eigenvalues of the Robin Laplacian when the Robin parameter goes to $-\infty$. The two first terms in the expansion have been obtained by K. Pankrashkin in the $2 D$-case and by K. Pankrashkin and N. Popoff in higher dimensions. The asymptotics display the influence of the curvature and the splitting between every two consecutive eigenvalues. The analysis is based on the approach developed by Fournais-Helffer for the semi-classical magnetic Laplacian. We also propose a WKB construction as candidate for the ground state energy.
\end{abstract}

\section{INTRODUCTION}

Let $\Omega \subset \mathbb{R}^{2}$ be an open domain with a smooth $C^{\infty}$ and compact boundary $\Gamma=\partial \Omega$ such that the boundary $\Gamma$ has a finite number of connected components. The unit outward normal vector of the boundary $\partial \Omega$ is denoted by $\nu$.

In this paper, we study the low-lying eigenvalues of the Robin Laplacian in $L^{2}(\Omega)$ with a large parameter. This is the operator

$$
\mathcal{P}^{\gamma}=-\Delta \text { in } L^{2}(\Omega),
$$

with domain,

$$
D\left(\mathcal{P}^{\gamma}\right)=\left\{u \in H^{2}(\Omega): \nu \cdot \nabla u+\gamma u=0 \quad \text { on } \partial \Omega\right\},
$$

where $\gamma<0$ is a given parameter. The operator $\mathcal{P}^{\gamma}$ is defined by the Friedrichs Theorem via the closed semi-bounded quadratic form, defined on $H^{1}(\Omega)$ by

$$
u \mapsto \mathcal{Q}^{\gamma}(u):=\|\nabla u\|_{L^{2}(\Omega)}^{2}+\gamma \int_{\partial \Omega}|u(x)|^{2} d s(x) .
$$

Denote by $\left(\lambda_{n}(\gamma)\right)$ the sequence of min-max values of the operator $\mathcal{P}^{\gamma}$. In [15, 16, 3], it is proved that, for every fixed $n \in \mathbb{N}$,

$$
\lambda_{n}(\gamma)=-\gamma^{2}+\kappa_{\max } \gamma+\gamma o(1) \quad \text { as } \gamma \rightarrow-\infty,
$$

where $\kappa_{\max }$ is the maximal curvature along the boundary $\Gamma$. Note that the first term in (1.4) was obtained previously (see [13] and references therein).

If the domain $\Omega$ is an exterior domain, then the operator $\mathcal{P}^{\gamma}$ has an essential spectrum; the essential spectrum is $[0, \infty)$, see e.g. [9]. In this case, the asymptotics in (1.4) show that, for every fixed $n$, if $-\gamma$ is selected sufficiently large, then $\lambda_{n}(\gamma)$ is in the discrete spectrum of the operator $\mathcal{P}^{\gamma}$. When the domain $\Omega$ is an interior domain, i.e. bounded, then by Sobolev embedding, the operator $\mathcal{P}^{\gamma}$ is with compact resolvent and its spectrum is purely discrete.

The aim of this paper is to improve the asymptotic expansion in (1.4) and to give the leading term of the spectral gap $\lambda_{n+1}(\gamma)-\lambda_{n}(\gamma)$. We will do this under a generic assumption on the domain $\Omega$ that we describe below.

Suppose that the boundary $\partial \Omega$ is parameterized by arc-length and $\kappa$ is the curvature of $\partial \Omega$. Let $\kappa_{\max }$ denotes the maximal value of $\kappa$. We choose the arc-length parametrization of the boundary such that $\kappa(0)=\kappa_{\max }$. Throughout this paper, we suppose that:

$$
\text { Assumption }(A) \quad\left\{\begin{array}{l}
\kappa \text { attains its maximum } \kappa_{\max } \text { at a unique point; } \\
\text { the maximum is non-degenerate, i.e. } k_{2}:=-\kappa^{\prime \prime}(0)>0 .
\end{array}\right.
$$

The main result in this paper is: 
Theorem 1.1. For any positive $n$, there exists a sequence $\left(\beta_{j, n}\right)_{j>0}$, such that, for any positive $M \in \mathbb{N}$, the eigenvalue $\lambda_{n}(\gamma)$ has, as $\gamma \rightarrow-\infty$, the asymptotic expansion

$$
\lambda_{n}(\gamma)=-\gamma^{2}+\gamma \kappa_{\max }+(2 n-1) \sqrt{\frac{k_{2}}{2}}|\gamma|^{1 / 2}+\sum_{j=0}^{M} \beta_{j, n}|\gamma|^{\frac{-j}{2}}+|\gamma|^{\frac{-M}{2}} o(1) .
$$

Compared with the existing semi-classics of Schrödinger operators (see [8]), the curvature in Theorem 1.1 acts as a potential well. The assumption $(A)$ indicates the case of a unique well. In the case considered here, one can say that, in the limit $\gamma \rightarrow-\infty$, the bottom of the spectrum of the operator $\mathcal{P}^{\gamma}$ is determined by the (boundary) operator $H^{\text {bnd }}:=-\frac{d^{2}}{d s^{2}}-\gamma^{2}+\gamma \kappa(s)$. Here $s$ is the arc-length parameterization of the boundary and the error through this approxomation is $\mathcal{O}(1)$. Loosely speaking, the reduced (boundary) operator $H^{\text {bnd }}$ is analyzed by replacing the potential (curvature) $-\kappa(s)$ by its quadratic approximation at the (unique) point of minimum.

As in [8], a natural and interesting question is to discuss the case of multiple wells. If we replace $(A)$ by

$$
\left(A^{\prime}\right)\left\{\begin{array}{l}
\kappa \text { attains its maximum } \kappa_{\max } \text { at a finite number of points }\left\{s_{0}, s_{1}, \cdots, s_{j}\right\} ; \\
\text { the maximum is non-degenerate, i.e. for all } i, k_{2, i}:=-\kappa^{\prime \prime}\left(s_{i}\right)>0 .
\end{array}\right.
$$

then many effects can appear depending on the values of the $\kappa^{\prime \prime}\left(s_{i}\right)$ (as in the case of the Schrödinger operator). In case of symmetries, the determination of the tunneling effect between the points of maximal curvature is expected to play an important role. A limiting situation is discussed in [7] when the domain $\Omega$ has two congruent corners (at a corner, we can assign the value $\infty$ to $\kappa_{\max }$ ). In the regular case (typically an ellipse), an interesting step could be a construction of WKB solutions in the spirit of a recent work by V. Bonnaillie, F. Hérau and N. Raymond [1. We address this WKB construction shortly in Section 9, and hope to come back at this point in a future work.

Acknowledgements. The authors would like to thank K. Pankrashkin for useful discussions. The first author is supported by the ANR programme NOSEVOL and the second author is supported by a grant from Lebanese University.

\section{TRANSFORMATION INTO A SEMI-CLASSICAL PROBLEM}

We will prove Theorem 1.1 by transforming it into a semi-classical problem as follows. Let

$$
h=\gamma^{-2} \text {. }
$$

The limit $\gamma \rightarrow-\infty$ is now equivalent to the semi-classical limit $h \rightarrow 0_{+}$. Notice the simple identity

$$
\forall u \in H^{1}(\Omega), \quad \mathcal{Q}^{\gamma}(u)=h^{-2}\left(\int_{\Omega}|h \nabla u|^{2}-h^{3 / 2} \int_{\partial \Omega}|u|^{2} d s(x)\right) .
$$

We define the operator

$$
\mathcal{L}_{h}=-h^{2} \Delta
$$

with domain,

$$
D\left(\mathcal{L}_{h}\right)=\left\{u \in H^{2}(\Omega): \nu \cdot h^{1 / 2} \nabla u-u=0 \text { on } \partial \Omega\right\} .
$$

Clearly,

$$
\sigma\left(\mathcal{P}^{\gamma}\right)=h^{-2} \sigma\left(\mathcal{L}_{h}\right)
$$

Let $\left(\mu_{n}(h)\right)$ be the sequence of min-max values of the operator $\mathcal{L}_{h}$. To determine the asymptotics of $\lambda_{n}(\gamma)$ as $\gamma \rightarrow-\infty$, we will determine the semi-classical asymptotics of $\mu_{n}(h)$ as $h \rightarrow 0_{+}$.

Now, Theorem 1.1 is a rephrasing of: 
Theorem 2.1. For any positive $n$, there exists a sequence $\left(\beta_{j, n}\right)_{j \geq 0}$, such that, as $h \rightarrow 0_{+}$, the eigenvalue $\mu_{n}(h)$ has for any positive $M \in \mathbb{N}$ the asymptotic expansion

$$
\mu_{n}(h)=-h-\kappa_{\max } h^{3 / 2}+(2 n-1) \sqrt{\frac{k_{2}}{2}} h^{7 / 4}+h^{2} \sum_{j=0}^{M} \beta_{j, n} h^{j / 4}+h^{2+\frac{M}{4}} o(1) .
$$

The proof of Theorem 2.1 consists of two major steps. In the first step, we establish a threeterm asymptotics

$$
\mu_{n}(h)=-h-\kappa_{\max } h^{3 / 2}+(2 n-1) \sqrt{\frac{k_{2}}{2}} h^{7 / 4}+h^{7 / 4} o(1),
$$

which is valid under the weaker assumption that the boundary of the domain $\Omega$ is $C^{4}$ smooth. This asymptotic expansion will be established in Sections 6 and 8 .

The next step is to construct good trial states and use the spectral theorem to establish existence of $n$-eigenvalues of the operator $\mathcal{L}_{h}$ satisfying the refined asymptotics

$$
\widetilde{\mu}_{n}(h)=-h-\kappa_{\max } h^{3 / 2}+(2 n-1) \sqrt{\frac{k_{2}}{2}} h^{7 / 4}+h^{15 / 8} \sum_{j=0}^{M} \zeta_{j, n} h^{j / 8}+h^{\frac{M+15}{8}} o(1),
$$

which in light of the three-term asymptotics for $\mu_{n}(h)$, yields the equality

$$
\mu_{n}(h)=\widetilde{\mu}_{n}(h),
$$

for $h>0$ sufficiently small. This analysis is presented in Section 7 . Right after the construction of the sequence $\left(\zeta_{j, n}\right)$, we will prove in Section 7.3 that $\zeta_{j, n}=0$ when $j$ is even. The coefficients in Theorem 1.1 are given by the formula $\beta_{j, n}=\zeta_{2 j+1, n}$.

Throughout this paper, the notation $\mathcal{O}\left(h^{\infty}\right)$ indicates a quantity satisfying that, for all $N \in \mathbb{N}$, there exists $C_{N}>0$ and $h_{N}>0$ such that, for all $h \in\left(0, h_{N}\right),\left|\mathcal{O}\left(h^{\infty}\right)\right| \leq C_{N} h^{N}$. Examples of such quantities are exponentially small quantities, like $\exp \left(-h^{-1 / 2}\right)$. The letter $C$ denotes a positive constant independent of the parameter $h$. The value of $C$ might change from one formula to another.

\section{BOUNDARY COORDINATES}

The key to prove Theorem 2.1 is a reduction to the boundary. Near the boundary, we do the computations using specific coordinates displaying the arc-length along the boundary and the normal distance to the boundary. In this section, we introduce the necessary notation to use these coordinates.

We will work in a connected component of the boundary. For simplicity, we suppose that $\Omega$ is simply connected; if $\Omega$ is not simply connected, we use the coordinates in each connected component independently. Let

$$
\mathbb{R} /(|\partial \Omega| \mathbb{Z}) \ni s \mapsto M(s) \in \partial \Omega
$$

be a parametrization of $\partial \Omega$. The unit tangent vector of $\partial \Omega$ at the point $M(s)$ of the boundary is given by

$$
T(s):=M^{\prime}(s)
$$

We define the curvature $\kappa(s)$ by the following identity

$$
T^{\prime}(s)=\kappa(s) \nu(s),
$$

where $\nu(s)$ is the unit vector, normal to to the boundary, pointing outward at the point $M(s)$. We choose the orientation of the parametrization $M$ to be counterclockwise, so

$$
\operatorname{det}(T(s), \nu(s))=1, \quad \forall s \in \mathbb{R} /(|\partial \Omega| \mathbb{Z}) .
$$

For all $\delta>0$, define

$$
\mathcal{V}_{\delta}=\{x \in \Omega: \operatorname{dist}(x, \partial \Omega)<\delta\},
$$


The map $\Phi$ is defined as follows :

$$
\Phi: \mathbb{R} /(|\partial \Omega| \mathbb{Z}) \times\left(0, t_{0}\right) \ni(s, t) \mapsto x=M(s)-t \nu(s) \in \mathcal{V}_{t_{0}} .
$$

Alternatively, for $x \in \mathcal{V}_{t_{0}}$, one can write

$$
\Phi^{-1}(x):=(s(x), t(x)) \in \mathbb{R} /(|\partial \Omega| \mathbb{Z}) \times\left(0, t_{0}\right),
$$

where $t(x)=\operatorname{dist}(x, \partial \Omega)$ and $s(x) \in \mathbb{R} /(|\partial \Omega| \mathbb{Z})$ is the coordinate of the point $M(s(x)) \in \partial \Omega$ satisfying $\operatorname{dist}(x, \partial \Omega)=|x-M(s(x))|$.

The determinant of the Jacobian of the transformation $\Phi^{-1}$ is given by:

$$
a(s, t)=1-t \kappa(s) .
$$

For all $u \in L^{2}\left(\mathcal{V}_{\delta}\right)$, define the function

$$
\widetilde{u}(s, t):=u(\Phi(s, t)) .
$$

For all $u \in H^{1}\left(\mathcal{V}_{t_{0}}\right)$, we have, with $\widetilde{u}=u \circ \Phi$,

$$
\int_{\mathcal{V}_{t_{0}}}|\nabla u|^{2} d x=\int\left[(1-t \kappa(s))^{-2}\left|\partial_{s} \widetilde{u}\right|^{2}+\left|\partial_{t} \widetilde{u}\right|^{2}\right](1-t \kappa(s)) d s d t,
$$

and

$$
\int_{\mathcal{V}_{t_{0}}}|u|^{2} d x=\int|\widetilde{u}(s, t)|^{2}(1-t \kappa(s)) d s d t
$$

\section{Three AUXILIARY OPERATORS}

In this section, we introduce three reference $1 D$-operators and determine their spectra.

4.1. 1D Laplacian on the half line. As simplest model, we start with the operator

$$
\mathcal{H}_{0,0}:=-\frac{d^{2}}{d \tau^{2}} \text { in } L^{2}\left(\mathbb{R}_{+}\right)
$$

with domain

$$
\left\{u \in H^{2}\left(\mathbb{R}_{+}\right): u^{\prime}(0)=-u(0)\right\} .
$$

The spectrum of this operator is $\{-1\} \cup[0, \infty)$. This operator will appear in Sections 6 , 7 and 9

However, this operator has the drawback that it is not with compact resolvent. To overcome this issue, we consider this operator in a bounded interval $(0, L)$ with $L$ sufficiently big and Dirichlet condition at $\tau=L$. This operator has a compact resolvent, and we will have fair knowledge about the behavior of its spectrum in the limit $L \rightarrow \infty$.

\subsection{D Laplacian on an interval.}

Let $h>0$ and $\rho \in(0,1)$. Consider the self-adjoint operator

$$
\mathcal{H}_{0, h}=-\frac{d^{2}}{d \tau^{2}} \quad \text { in } L^{2}\left(\left(0, h^{-\rho}\right)\right)
$$

with domain,

$$
D\left(\mathcal{H}_{0, h}\right)=\left\{u \in H^{2}\left(\left(0, h^{-\rho}\right)\right): u^{\prime}(0)=-u(0) \quad \text { and } \quad u\left(h^{-\rho}\right)=0\right\} .
$$

The spectrum of the operator $\mathcal{H}_{0, h}$ is purely discrete and consists of a strictly increasing sequence of eigenvalues denoted by $\left(\lambda_{n}\left(\mathcal{H}_{0, h}\right)\right)$.

Note that this operator is associated with the quadratic form

$$
V_{h} \ni u \mapsto \int_{0}^{h^{-\rho}}\left|u^{\prime}(\tau)\right|^{2} d \tau-|u(0)|^{2},
$$

with $V_{h}:=\left\{v \in H^{1}\left(\left(0, h^{-\rho}\right)\right) \mid v\left(h^{-\rho}\right)=0\right\}$.

The next lemma gives the localization of the two first eigenvalues $\lambda_{1}\left(\mathcal{H}_{0, h}\right)$ and $\lambda_{2}\left(\mathcal{H}_{0, h}\right)$ for small values of $h$. 
Lemma 4.1. As $h \rightarrow 0_{+}$, there holds

$$
\lambda_{1}\left(\mathcal{H}_{0, h}\right)=-1+4(1+o(1)) \exp \left(-2 h^{-\rho}\right) \quad \text { and } \quad \lambda_{2}\left(\mathcal{H}_{0, h}\right) \geq 0 .
$$

Proof. Let $w \geq 0$ and $\lambda=-w^{2}$ be a negative eigenvalue of the operator $\mathcal{H}_{0, h}$ with an eigenfunction $u$. We have,

$$
-u^{\prime \prime}=\lambda u \quad \text { in }\left(0, h^{-\rho}\right), \quad u^{\prime}(0)=-u(0), \quad u\left(h^{-\rho}\right)=0 .
$$

If $w=0$ and $h<1$, then the unique solution of (4.6) is $u=0$. Thus, we suppose that $w>0$. Easy computations give us

$$
u(\tau)=A\left(\exp (-w \tau)-\exp \left(-2 w h^{-\rho}\right) \exp (w \tau)\right)
$$

with the following condition on $w$,

$$
w\left(1+\exp \left(-2 w h^{-\rho}\right)\right)=1-\exp \left(-2 w h^{-\rho}\right) .
$$

Define the function

$$
f(v)=v\left(1+\exp \left(-2 v h^{-\rho}\right)\right)-\left(1-\exp \left(-2 v h^{-\rho}\right)\right)=v-1+(v+1) \exp \left(-2 v h^{-\rho}\right) .
$$

We will locate the zeros of $f$ in $\mathbb{R}_{+}=(0, \infty)$. Notice that $f(v)>0$ for all $v \geq 1$. This forces the solution $w$ of (4.8) to live in $(0,1)$.

If $v \in\left[h^{\rho / 2}, 1-h^{\rho / 2}\right]$, then as $h \rightarrow 0_{+}$, the term $\exp \left(-2 v h^{-\rho}\right)$ is exponentially small and

$$
\frac{v+1}{v-1} \exp \left(-2 v h^{-\rho}\right) \rightarrow 0 \text {. }
$$

Writing

$$
f(v)=(v-1)\left(1+\frac{v+1}{v-1} \exp \left(-2 v h^{-\rho}\right)\right),
$$

we observe that there exists $h_{0} \in(0,1)$ such that, for all $h \in\left(0, h_{0}\right)$,

$$
f(v)<0 \quad \text { in }\left[h^{\rho / 2}, 1-h^{\rho / 2}\right] .
$$

This forces $w$ to live in $\left(0, h^{\rho / 2}\right) \cup\left(1-h^{\rho / 2}, 1\right)$. Since $f(v)=-1+o(1)$ as $v \rightarrow 0_{+}$, then there exists $h_{0} \in(0,1)$ such that, for all $h \in\left(0, h_{0}\right)$ and $v \in\left(0, h^{\rho / 2}\right), f(v)<0$. Thus, $f$ may vanish in $\left(1-h^{\rho / 2}, 1\right)$ only. Notice that

$$
f^{\prime}(v)=1-2 h^{-\rho}\left(v+1-\frac{h^{\rho}}{2}\right) \exp \left(-2 v h^{-\rho}\right)>0 \quad \text { in } \quad\left(1-h^{\rho / 2}, 1\right) .
$$

Thus $f$ has a unique zero $w_{h}$ in the interval $\left(1-h^{\rho / 2}, 1\right)$. This zero corresponds to the first eigenvalue of $\mathcal{H}_{0, h}$ by the relation $\lambda_{1}\left(\mathcal{H}_{0, h}\right)=-w_{h}^{2}$. Inserting

$$
w_{h}=1+\mathcal{O}\left(h^{\rho / 2}\right)
$$

into (4.8), we get that

$$
w_{h}=1-2(1+o(1)) \exp \left(-2 h^{-\rho}\right) .
$$

As a consequence, we observe that $\lambda_{1}\left(\mathcal{H}_{0, h}\right)=-w_{h}^{2}$ satisfies (4.5). Since $f$ does not vanish in $\mathbb{R}_{+} \backslash\left(1-h^{\rho / 2}, 1\right)$, then $\lambda_{2}\left(\mathcal{H}_{0, h}\right) \geq 0$.

Remark 4.2. Note that by domain monotonicity and minimax characterization, we get directly from the spectrum of $\mathcal{H}_{0,0}$ that $\lambda_{1}\left(\mathcal{H}_{0, h}\right) \geq-1$ and $\lambda_{2}\left(\mathcal{H}_{0, h}\right) \geq 0$.

Remark 4.3. Along the proof of Lemma 4.1, we obtain that the eigenspace of the first eigenvalue $\lambda_{1}\left(\mathcal{H}_{0, h}\right)$ is generated by the function

$$
u_{0, h}(\tau)=A_{h}\left(\exp \left(-w_{h} \tau\right)-\exp \left(-2 w_{h} h^{-\rho}\right) \exp \left(w_{h} \tau\right)\right)
$$


with $w_{h}$ a constant satisfying (4.9). The constant $A_{h}$ is selected so that $u_{0, h}$ is normalized in $L^{2}\left(\left(0, h^{-\rho}\right)\right)$. As $h \rightarrow 0_{+}, A_{h}$ satisfies,

\section{3. $1 \mathrm{D}$ operator in a weighted space.}

$$
A_{h}=\sqrt{2}+\mathcal{O}\left(h^{-\rho / 2} \exp \left(-h^{-\rho}\right)\right) .
$$

Let $h \in(0,1), \beta \in \mathbb{R}, \rho \in(0,1 / 2)$ and $|\beta| h^{\frac{1}{2}-\rho}<\frac{1}{3}$. Consider the self-adjoint operator

$$
\mathcal{H}_{\beta, h}=-\frac{d^{2}}{d \tau^{2}}+\beta h^{1 / 2}\left(1-\beta h^{1 / 2} \tau\right)^{-1} \frac{d}{d \tau} \quad \text { in } \quad L^{2}\left(\left(0, h^{-\rho}\right) ;\left(1-\beta h^{1 / 2} \tau\right) d \tau\right),
$$

with domain

$$
D\left(\mathcal{H}_{\beta, h}\right)=\left\{u \in H^{2}\left(\left(0, h^{-\rho}\right)\right): u^{\prime}(0)=-u(0) \text { and } u\left(h^{-\rho}\right)=0\right\} .
$$

The operator $\mathcal{H}_{\beta, h}$ is the Friedrichs extension in $L^{2}\left(\left(0, h^{-\rho}\right) ;\left(1-\beta h^{1 / 2} \tau\right) d \tau\right)$ associated with the quadratic form defined for $u \in V_{h}$, by

$$
q_{\beta, h}(u)=\int_{0}^{h^{-\rho}}\left|u^{\prime}(\tau)\right|^{2}\left(1-\beta h^{1 / 2} \tau\right) d \tau-|u(0)|^{2} .
$$

The operator $\mathcal{H}_{\beta, h}$ is with compact resolvent. The strictly increasing sequence of the eigenvalues of $\mathcal{H}_{\beta, h}$ is denoted by $\left(\lambda_{n}\left(\mathcal{H}_{\beta, h}\right)\right)_{n \in \mathbb{N}^{*}}$.

The next lemma localizes the spectrum of $\mathcal{H}_{\beta, h}$ near that of the operator $\mathcal{H}_{0, h}$ as $h$ goes to 0.

Lemma 4.4. There exist constants $C>0$ and $h_{0} \in(0,1)$ such that, for all $h \in\left(0, h_{0}\right)$ and $n \in \mathbb{N}^{*}$, there holds,

$$
\left|\lambda_{n}\left(\mathcal{H}_{\beta, h}\right)-\lambda_{n}\left(\mathcal{H}_{0, h}\right)\right| \leq C|\beta| h^{\frac{1}{2}-\rho}\left|\lambda_{n}\left(\mathcal{H}_{0, h}\right)\right| .
$$

Proof. There exists a constant $C>0$ such that, for all $u \in H^{1}\left(\left(0, h^{-\rho}\right)\right)$,

$$
\begin{aligned}
& \left|q_{\beta, h}(u)-q_{0, h}(u)\right| \leq|\beta| h^{\frac{1}{2}-\rho} q_{0, h}(u), \\
& \left|\|u\|_{L^{2}\left(\left(0, h^{-\rho}\right) ;\left(1-\beta h^{1 / 2} \tau\right) d \tau\right)}^{2}-\|u\|_{L^{2}\left(\left(0, h^{-\rho}\right) ; d \tau\right)}^{2}\right| \leq|\beta| h^{\frac{1}{2}-\rho}\|u\|_{L^{2}\left(\left(0, h^{-\rho}\right) ; d \tau\right)}^{2} .
\end{aligned}
$$

The conclusion of the lemma is now a simple application of the min-max principle.

The next proposition states a two-term asymptotic expansion of the eigenvalue $\lambda_{1}\left(\mathcal{H}_{\beta, h}\right)$ as $h \rightarrow 0_{+}$.

Proposition 4.5. There exist constants $C>0$ and $h_{0} \in(0,1)$ such that, for all $h \in\left(0, h_{0}\right)$ and $|\beta| h^{\rho}<\frac{1}{3}$, there holds,

$$
\left|\lambda_{1}\left(\mathcal{H}_{\beta, h}\right)-\left(-1-\beta h^{1 / 2}\right)\right| \leq C \beta^{2} h .
$$

Proof. Consider the function

$$
f(\tau)=\chi\left(\tau h^{\rho}\right) u_{0}(\tau)
$$

where

and $\chi \in C_{c}^{\infty}([0, \infty))$ satisfies

$$
u_{0}(\tau)=\sqrt{2} \exp (-\tau)
$$

$$
0 \leq \chi \leq 1 \text { in }[0, \infty), \quad \chi=1 \text { in }[0,1 / 2) \text { and } \quad \chi=0 \text { in }[1 / 2, \infty) .
$$

Clearly, the function $f$ is in the domain of the operator $\mathcal{H}_{\beta, h}$. It is easy to get the estimates:

$$
\begin{aligned}
& \left|\|f\|_{L^{2}\left(\left(0, h^{-\rho}\right) ;\left(1-\beta h^{1 / 2} \tau\right) d \tau\right)}^{2}-1\right| \leq C \beta^{2} h, \\
& \left\|\left\{\mathcal{H}_{\beta, h}-\left(-1-\beta h^{1 / 2}\right)\right\} f\right\|_{L^{2}\left(\left(0, h^{-\rho}\right) ;\left(1-\beta h^{1 / 2} \tau\right) d \tau\right)} \leq C \beta^{2} h .
\end{aligned}
$$

By the spectral theorem, we deduce that there exists an eigenvalue $\lambda\left(\mathcal{H}_{\beta, h}\right)$ of $\mathcal{H}_{\beta, h}$ such that

$$
\left|\lambda\left(\mathcal{H}_{\beta, h}\right)-\left(-1-\beta h^{1 / 2}\right)\right| \leq C \beta^{2} h .
$$


Thanks to Lemmas 4.1 and 4.4, we have

$$
\lambda_{1}\left(\mathcal{H}_{\beta, h}\right)=\lambda\left(\mathcal{H}_{\beta, h}\right) .
$$

\section{LOCALIZATION OF GROUND STATES}

\subsection{Localization near the boundary.}

Theorem 5.1. Let $M \in(-1,0)$. For all $\alpha<\sqrt{-M}$, there exist constants $C>0$ and $h_{0} \in(0,1)$ such that, if $u_{h}$ is a normalized eigenfunction of $\mathcal{L}_{h}$ with eigenvalue $\mu(h) \leq M h$, then, for all $h \in\left(0, h_{0}\right)$,

$$
\int_{\Omega}\left(\left|u_{h}(x)\right|^{2}+h\left|\nabla u_{h}(x)\right|^{2}\right) \exp \left(\frac{2 \alpha \operatorname{dist}(x, \partial \Omega)}{h^{1 / 2}}\right) d x \leq C .
$$

Proof. Let $t(x)=\operatorname{dist}(x, \partial \Omega)$ and $\Phi(x)=\exp \left(\frac{\alpha t(x)}{h^{1 / 2}}\right)$. We have the simple identity

$$
\left\langle\Phi^{2} u_{h}, \mathcal{L}_{h} u_{h}\right\rangle_{L^{2}(\Omega)}=\mu(h)\left\|\Phi u_{h}\right\|_{L^{2}(\Omega)}^{2} .
$$

By an integration by parts, we get the useful identity

$$
q_{h}^{\Phi}\left(u_{h}\right):=\int_{\Omega}\left(\left|h \nabla\left(\Phi u_{h}\right)\right|^{2}-h^{2}|\nabla \Phi|^{2}\left|u_{h}\right|^{2}\right) d x-h^{3 / 2} \int_{\partial \Omega}\left|\Phi u_{h}\right|^{2} d s(x)=\mu(h)\left\|\Phi u_{h}\right\|_{L^{2}(\Omega)}^{2} .
$$

Consider a partition of unity of $\mathbb{R}$

$$
\chi_{1}^{2}+\chi_{2}^{2}=1
$$

such that $\chi_{1}=1$ in $(-\infty, 1)$, supp $\chi_{1} \subset(-\infty, 2), \chi_{1} \geq 0$ and $\chi_{2} \geq 0$ in $\mathbb{R}$.

Define

$$
\chi_{j, h}(x)=\chi_{j}\left(\frac{t(x)}{h^{1 / 2}}\right), \quad j \in\{1,2\} .
$$

Associated with this partition of unity, we have the simple standard decomposition

$$
q_{h}^{\Phi}\left(u_{h}\right)=\sum_{j=1}^{2} q_{j, h}^{\Phi}\left(u_{h}\right),
$$

where

$$
q_{1, h}^{\Phi}\left(u_{h}\right)=\int_{\Omega}\left(\left|h \nabla\left(\chi_{1, h} \Phi u_{h}\right)\right|^{2}-h^{2}\left|\nabla\left(\chi_{1, h} \Phi\right)\right|^{2}\left|u_{h}\right|^{2}\right) d x-h^{3 / 2} \int_{\partial \Omega}\left|\chi_{1, h} \Phi u_{h}\right|^{2} d s(x),
$$

and

$$
q_{2, h}^{\Phi}\left(u_{h}\right)=\int_{\Omega}\left(\left|h \nabla\left(\chi_{2, h} \Phi u_{h}\right)\right|^{2}-h^{2}\left|\nabla\left(\chi_{2, h} \Phi\right)\right|^{2}\left|u_{h}\right|^{2}\right) d x
$$

A rearrangement of the terms in (5.1) - (5.3) will yield the existence of a constant $C$ such that

$$
\begin{array}{r}
\int_{\left\{t(x) \leq 2 h^{1 / 2}\right\}}\left(\left|h \nabla\left(\chi_{1, h} \Phi u_{h}\right)\right|^{2}-C h\left|\Phi u_{h}\right|^{2}-\mu(h)\left|\chi_{1, h} \Phi u_{h}\right|^{2}\right) d x-h^{3 / 2} \int_{\partial \Omega}\left|\chi_{1, h} \Phi u_{h}\right|^{2} d s(x) \\
\leq \int_{\Omega}\left(-\left|h \nabla\left(\chi_{2, h} \Phi u_{h}\right)\right|^{2}+\left(M+\alpha^{2}\right) h\left|\chi_{2, h} \Phi u_{h}\right|^{2}\right) d x .
\end{array}
$$

The definition of $\Phi$, the assumption on $\mu(h)<0$, and the normalization of $u_{h}$ yield

$$
-\int_{\left\{t(x) \leq 2 h^{1 / 2}\right\}} \mu(h)\left|\chi_{1, h} \Phi u_{h}\right|^{2} d x \geq 0,
$$

and (notice that $0 \leq \Phi(x) \leq \exp (2 \alpha)$ when $t(x) \leq 2 h^{1 / 2}$ )

$$
-\int_{\left\{t(x) \leq 2 h^{1 / 2}\right\}} h\left|\Phi u_{h}\right|^{2} \geq-2 C h .
$$


At this stage we are left with the inequality:

$$
\begin{aligned}
\int_{\left\{t(x) \leq 2 h^{1 / 2}\right\}}\left(\mid h \nabla\left(\chi_{1, h} \Phi\right.\right. & \left.\left.u_{h}\right)\left.\right|^{2}\right) d x-h^{3 / 2} \int_{\partial \Omega}\left|\chi_{1, h} \Phi u_{h}\right|^{2} d s(x) \\
& \leq \int_{\Omega}\left(-\left|h \nabla\left(\chi_{2, h} \Phi u_{h}\right)\right|^{2}+\left(M+\alpha^{2}\right) h\left|\chi_{2, h} \Phi u_{h}\right|^{2}\right) d x+2 C h .
\end{aligned}
$$

In a small tubular neighborhood of the boundary, we may use the boundary coordinates $(s, t)$ recalled in Section 3 and obtain

$$
\begin{aligned}
& \int_{\left\{t(x) \leq 2 h^{1 / 2}\right\}}\left|h \nabla\left(\chi_{1, h} \Phi u_{h}\right)\right|^{2} d x-h^{3 / 2} \int_{\partial \Omega}\left|\chi_{1, h} \Phi u_{h}\right|^{2} d s(x) \\
& \geq\left(1-C h^{1 / 2}\right) \int_{-|\partial \Omega| / 2}^{|\partial \Omega| / 2}\left(\int_{0}^{2 h^{1 / 2}} h^{2}\left|\partial_{t}\left(\chi_{1, h} \Phi u_{h}\right)\right|^{2} d t-h^{3 / 2}\left|\left(\chi_{1, h} \Phi u_{h}\right)(s, t=0)\right|^{2}\right) d s .
\end{aligned}
$$

Let $\frac{1}{2}<\rho<1$. We do the change of variable $\tau=h^{-1} t$ and introduce the function $v(s, \tau)=$ $\left(\chi_{1, h} \Phi u_{h}\right)(s, t)$. The function $v$ vanishes for $\tau \geq 2 h^{-1 / 2}$, and when $h$ is sufficiently small, $2 h^{-1 / 2} \leq h^{-\rho}$. In this way we get

$\int_{0}^{2 h^{1 / 2}} h^{2}\left|\partial_{t}\left(\chi_{1, h} \Phi u_{h}\right)\right|^{2} d t-h^{3 / 2}\left|\left(\chi_{1, h} \Phi u_{h}\right)(s, t=0)\right|^{2}=h\left(\int_{0}^{h^{-\rho}}\left|\partial_{\tau} v\right|^{2} d \tau-h^{1 / 2}|v(s, \tau=0)|^{2}\right)$.

Now, we use the min-max principle and Lemma 4.1 to obtain

$$
\int_{0}^{h^{-\rho}}\left|\partial_{\tau} v\right|^{2} d \tau-h^{1 / 2}|v(s, \tau=0)|^{2} \geq \lambda_{1}\left(\mathcal{H}_{0, h}\right) \int_{0}^{h^{-\rho}}|v|^{2} d \tau \geq-2 h \int_{0}^{h^{-\rho}}|v|^{2} d \tau .
$$

Coming back to the $t$ variable, we get

$$
\int_{0}^{2 h^{1 / 2}} h^{2}\left|\partial_{t}\left(\chi_{1, h} \Phi u_{h}\right)\right|^{2} d t-h^{3 / 2}\left|\left(\chi_{1, h} \Phi u_{h}\right)(s, t=0)\right|^{2} \geq-2 h \int_{0}^{2 h^{1 / 2}} h^{2}\left|\partial_{t}\left(\chi_{1, h} \Phi u_{h}\right)\right|^{2} d t .
$$

Inserting this into (5.6), we obtain

$$
\begin{aligned}
& \int_{\left\{t(x) \leq 2 h^{1 / 2}\right\}}\left|h \nabla\left(\chi_{1, h} \Phi u_{h}\right)\right|^{2} d x-h^{3 / 2} \int_{\partial \Omega}\left|\chi_{1, h} \Phi u_{h}\right|^{2} d s(x) \\
& \quad \geq-2\left(1-C h^{1 / 2}\right) h \int_{-|\partial \Omega| / 2}^{|\partial \Omega| / 2} \int_{0}^{2 h^{1 / 2}}\left|\chi_{1, h} \Phi u_{h}\right|^{2} d t d s \geq-C^{\prime} h \int_{\Omega}\left|\chi_{1, h} \Phi u_{h}\right|^{2} d x \geq-C^{\prime} h .
\end{aligned}
$$

Now, we infer from (5.5)

$$
-\left(C^{\prime}+2 C\right) h \geq \int_{\Omega}\left(-\left|h \nabla\left(\chi_{2, h} \Phi u_{h}\right)\right|^{2}+\left(M+\alpha^{2}\right) h\left|\chi_{2, h} \Phi u_{h}\right|^{2}\right) d x .
$$

Since $\left(M+\alpha^{2}\right)<0$, we deduce that

$$
\int_{\Omega}\left(\left|\nabla\left(\chi_{2, h} \Phi u_{h}\right)\right|^{2}-\left(M+\alpha^{2}\right)\left|\chi_{2, h} \Phi u_{h}\right|^{2}\right) d x \leq\left(C^{\prime}+2 C\right),
$$

which is enough to deduce the conclusion in Theorem 5.1 . 


\subsection{Localization near the point of maximal curvature.}

We will apply the result in Lemma 4.5 to obtain a lower bound of the quadratic form

$$
q_{h}(u)=\int|h \nabla u|^{2} d x-h^{3 / 2} \int_{\partial \Omega}|u|^{2} d s(x) .
$$

Theorem 5.2. There exist constants $C>0$ and $h_{0} \in(0,1)$ such that, for all $h \in\left(0, h_{0}\right)$ and $u \in H^{1}(\Omega)$,

where

$$
q_{h}(u) \geq \int_{\Omega} U_{h}(x)|u(x)|^{2} d x,
$$

$$
U_{h}(x)= \begin{cases}0 & \text { if } \operatorname{dist}(x, \partial \Omega)>2 h^{1 / 8}, \\ -h-\kappa(s(x)) h^{3 / 2}-C h^{7 / 4} & \text { if } \operatorname{dist}(x, \partial \Omega) \leq 2 h^{1 / 8} .\end{cases}
$$

Proof. Consider a partition of unity of $\mathbb{R}$,

$$
\chi_{1}^{2}+\chi_{2}^{2}=1
$$

with $\chi_{1}=1$ in $(-\infty, 1]$ and $\operatorname{supp} \chi_{2} \subset[1, \infty)$. For $j \in\{1,2\}$, put,

$$
\chi_{j, h}(x)=\chi_{j}\left(\frac{\operatorname{dist}(x, \partial \Omega)}{h^{1 / 8}}\right) .
$$

We have the decomposition

$$
q_{h}(u)=q_{1, h}(u)+q_{2, h}(u)-h^{2} \sum_{j=1}^{2}\left\|\left|\nabla \chi_{j, h}\right| u\right\|_{L^{2}(\Omega)}^{2},
$$

where

$$
\begin{gathered}
q_{1, h}(u)=\int_{\Omega}\left|h \nabla\left(\chi_{1, h} u\right)\right|^{2} d x-h^{3 / 2} \int_{\partial \Omega}|u|^{2} d s(x), \\
q_{2, h}(u)=\int_{\Omega}\left|h \nabla\left(\chi_{2, h} u\right)\right|^{2} d x \geq 0
\end{gathered}
$$

and

Thus,

$$
h^{2}\left\|\left|\nabla \chi_{j, h}\right| u\right\|_{L^{2}(\Omega)}^{2} \leq C_{1} h^{7 / 4} \int_{\left\{\operatorname{dist}(x, \partial \Omega) \leq 2 h^{1 / 8}\right\}}|u|^{2} d x .
$$

$$
q_{h}(u) \geq q_{1, h}\left(\chi_{1, h} u\right)-C_{1} h^{7 / 4} \int_{\left\{\operatorname{dist}(x, \partial \Omega) \leq 2 h^{1 / 8}\right\}}|u|^{2} d x .
$$

In the support of $\chi_{1, h}$, we may use the boundary coordinates $(s, t)$ recalled in Section 3 and write

$$
\begin{aligned}
q_{1, h}(u) \geq \int_{-|\partial \Omega| / 2}^{|\partial \Omega| / 2}\left(\int_{0}^{2 h^{1 / 8}}\left|h \partial_{t}\left(\chi_{1, h} u\right)\right|^{2}(1-\right. & \left.\kappa(s) t) d t-h^{3 / 2}\left|\left(\chi_{1, h} u\right)(s, t=0)\right|^{2}\right) d s \\
& +\int_{-|\partial \Omega| / 2}^{|\partial \Omega| / 2}\left|\partial_{s}\left(\chi_{1, h} u\right)\right|^{2}(1-\kappa(s) t)^{-1} d t d s .
\end{aligned}
$$

Recall the operator in $\mathcal{H}_{\beta, h}$ in (4.12). By a simple scaling argument (similar to the one appearing after (5.6)) and the min-max principle, we have

$$
\begin{aligned}
\int_{0}^{2 h^{1 / 8}}\left|h \partial_{t}\left(\chi_{1, h} u\right)\right|^{2}(1-\kappa(s) t) d t-h^{3 / 2} \mid & \left.\left(\chi_{1, h} u\right)(s, t=0)\right|^{2} \\
& \geq h \lambda_{1}\left(\mathcal{H}_{\kappa(s), h}\right) \int_{0}^{h^{1 / 8}}\left|\chi_{1, h} u\right|^{2}(1-\kappa(s) t) d t .
\end{aligned}
$$


Thanks to Proposition 4.5, we deduce the following lower bound,

$$
q_{1, h}(u) \geq \int_{\Omega}\left(-h-\kappa(s(x)) h^{3 / 2}-C_{2} h^{2}\right)\left|\chi_{1, h} u\right|^{2} d x .
$$

Therefore, after collecting the lower bounds for $q_{1, h}(u)$ and $q_{2, h}(u)$, we deduce that

$$
\begin{aligned}
q_{h}(u) & \geq \int_{\Omega}\left(-h-\kappa(s(x)) h^{3 / 2}-C_{2} h^{2}\right)\left|\chi_{1, h} u\right|^{2} d x-C_{1} h^{7 / 4} \int_{\left\{\operatorname{dist}(x, \partial \Omega) \leq 2 h^{1 / 8}\right\}}|u|^{2} d x \\
& \geq \int_{\left\{\operatorname{dist}(x, \partial \Omega) \leq 2 h^{1 / 8}\right\}}\left(-h-\kappa(s(x)) h^{3 / 2}-C_{1} h^{7 / 4}-C_{2} h^{2}\right)|u|^{2} d x .
\end{aligned}
$$

To finish the proof of Theorem [5.2, we select the constant $C=C_{1}+C_{2}$ in the definition of $U_{h}$.

Remark 5.3. Let $M>0$ and $u_{h}$ a normalized eigenfunction of $\mathcal{L}_{h}$ with eigenvalue satisfying $\mu(h) \leq-h-h^{3 / 2} \kappa_{\max }+M h^{7 / 4}$. Using the inequalities in (5.8)-(5.9) and the upper bound satisfied by $\mu(h)$, we get the interesting estimate

$$
\left\|\partial_{s}\left(\chi_{1, h} u_{h}\right)\right\|_{2} \leq C h^{-1 / 8} .
$$

To see this, notice that $q_{1, h}\left(u_{h}\right) \leq q_{h}\left(u_{h}\right) \leq \mu(h) \leq-h-h^{3 / 2} \kappa_{\max }+M h^{7 / 4}$. Now plugging (5.10) into (5.8), we get

$$
\int_{-|\partial \Omega| / 2}^{|\partial \Omega| / 2}\left|h \partial_{s}\left(\chi_{1, h} u\right)\right|^{2}(1-\kappa(s) t)^{-1} d t d s \leq C\left(\mu(h)-\left(-h-h^{3 / 2} \kappa_{\max }-C_{2} h^{2}\right)\right) \leq C h^{7 / 8} .
$$

In the support of $\chi_{1, h}$, we may write $(1-t \kappa(s))^{-1} \geq 1$ and get the inequality in (5.11).

Remark 5.4. The min-max principle and the conclusion in Theorem 5.2 yield a lower bound on the first eigenvalue of the operator $\mathcal{L}_{h}$, namely, there exists $h_{0}>0$ such that, for all $h \in\left(0, h_{0}\right)$,

$$
\mu_{1}(h) \geq-h-\kappa_{\max } h^{3 / 2}-C h^{7 / 4} .
$$

As a consequence of Theorem 5.2, we can prove, following the same steps as in [5, Thm. 4.9], that:

Theorem 5.5. Let $M>0$ and $\chi_{1} \in C_{c}^{\infty}\left(\overline{\mathbb{R}_{+}}\right)$. There exist constants $C>0, \alpha>0$ and $h_{0} \in(0,1)$ such that, if $u_{h}$ is a normalized eigenfunction of the operator $\mathcal{L}_{h}$ with eigenfunction $\mu(h) \leq-h-\kappa_{\max } h^{3 / 2}+M h^{7 / 4}$, then for all $h \in\left(0, h_{0}\right)$,

$$
\int_{\Omega} \chi_{1}\left(\frac{\operatorname{dist}(x, \partial \Omega)}{h^{1 / 8}}\right)^{2}\left\{\left|u_{h}(x)\right|^{2}+h\left|\nabla u_{h}(x)\right|^{2}\right\} \exp \left(\frac{2 \alpha|s(x)|^{2}}{h^{1 / 4}}\right) d x \leq C .
$$

The localization of the bound states in Theorem 5.5 near the set

$$
n(\partial \Omega)=\left\{x \in \partial \Omega: \kappa(s(x))=\kappa_{\max }\right\}
$$

can be expressed using the Agmon distance $\hat{d}_{\partial \Omega}(s, n(\partial \Omega))$ in $\partial \Omega$ associated with the metric $\left(\kappa_{\max }-\kappa(s)\right) d s^{2}$. Let us define

$$
\hat{d}(x, n(\partial \Omega), h)=\hat{d}_{\partial \Omega}(s(x), n(\partial \Omega)) \chi_{1}(d(x, \partial \Omega))+h^{-1 / 4} d(x, \partial \Omega) .
$$

As in [4, Theorem 8.3.4], Theorem [5.2 yields the following conclusion. Let $M>0$. There exists $\delta>0$ and for all $\epsilon>0$, there exist $h_{\epsilon}>0$ and $C_{\epsilon}>0$ such that, for all $h \in\left(0, h_{\epsilon}\right]$, if $u_{h}$ is a normalized ground state as in Theorem 5.5, then,

$$
\left\|\exp \left(\delta h^{-1 / 4} \hat{d}(x, n(\partial \Omega), h)\right) u_{h}\right\|_{L^{2}(\Omega)} \leq C_{\epsilon} \exp \left(\epsilon h^{-1 / 4}\right)
$$

This estimate may have an advantage over that in Theorem 5.5 when approximating the bound states by suitably constructed trial states.

A simple consequence of Theorems 5.1 and 5.5 is: 
Corollary 5.6. Let $\chi \in C_{c}^{\infty}\left(\overline{\mathbb{R}_{+}}\right)$such that $\operatorname{supp} \chi \subset\left[0, t_{0}\right)$. Under the assumptions in Theorem 5.5, if $t_{0}$ is sufficiently small, then there exists a constant $C$ such that,

$$
\int_{\Omega}|s(x)|^{k}|\chi(t(x))|^{2}\left|u_{h}(x)\right|^{2} d x \leq C h^{k / 8} .
$$

Here we have also used the first localization.

\section{Construction of approximate eigenfunctions}

In this section, we construct trial states $\left(\phi_{n}\right)$ in the domain of the operator $\mathcal{L}_{h}$ such that, for every fixed $n \in \mathbb{N}$, we have

$$
\left\|\mathcal{L}_{h} \phi_{n}-\left(-h-\kappa_{\max } h^{3 / 2}+(2 n-1) \sqrt{\frac{-\kappa^{\prime \prime}(0)}{2}} h^{7 / 4}\right) \phi_{n}\right\|_{L^{2}(\Omega)} \leq C h^{15 / 8}\left\|\phi_{n}\right\|_{L^{2}(\Omega)} .
$$

Notice that a direct consequence of (6.1) is:

$$
\left\langle\mathcal{L}_{h} \phi_{n}-\left(-h-\kappa_{\max } h^{3 / 2}+(2 n-1) \sqrt{\frac{-\kappa^{\prime \prime}(0)}{2}} h^{7 / 4}\right) \phi_{n}, \phi_{n}\right\rangle_{L^{2}(\Omega)} \leq C h^{15 / 8}\left\|\phi_{n}\right\|_{L^{2}(\Omega)}^{2} .
$$

The trial states $\left(\phi_{n}\right)$ will be supported near the boundary of $\Omega$, so that the use of the boundary coordinates $(s, t)$ recalled in Section 3 is valid. The operator $\mathcal{L}_{h}$ is expressed in $(s, t)$ coordinates as follows:

$$
\mathcal{L}_{h}=-h^{2} a^{-1} \partial_{s}\left(a^{-1} \partial_{s}\right)-h^{2} a^{-1} \partial_{t}\left(a \partial_{t}\right) \quad\left(\text { in } L^{2}(a d s d t)\right),
$$

where

$$
a(s, t)=1-t \kappa(s) .
$$

In boundary coordinates, the Robin condition in (1.2) becomes

$$
h \partial_{t} u=-h^{3 / 2} u \quad \text { on } \quad t=0 .
$$

The change of variable $(s, t) \mapsto\left(h^{1 / 8} \sigma, h^{1 / 2} \tau\right)$ transforms the above expression of $\mathcal{L}_{h}$ to

$$
\mathcal{L}_{h}=h\left(-h^{3 / 4} \tilde{a}^{-1} \partial_{\sigma}\left(\tilde{a}^{-1} \partial_{\sigma}\right)-\tilde{a}^{-1} \partial_{\tau}\left(\tilde{a} \partial_{\tau}\right)\right)
$$

where

$$
\tilde{a}(\sigma, \tau)=1-h^{1 / 2} \tau \kappa\left(h^{1 / 8} \sigma\right) .
$$

The boundary condition is now transformed to

$$
\partial_{\tau} \widetilde{u}=-\widetilde{u} \quad \text { on } \quad \tau=0 .
$$

Recall that the value of the maximal curvature is $\kappa_{\max }=\kappa(0)$. We have the following asymptotic expansions:

$$
\begin{aligned}
& \tilde{a}(\sigma, \tau)=1-h^{1 / 2} \tau \kappa(0)+h^{1 / 2} \tau \epsilon_{1}(\sigma), \\
& \tilde{a}^{-1} \partial_{\tau} \widetilde{a}(\sigma, \tau)=-h^{1 / 2} \kappa(0)-h^{3 / 4} \frac{\kappa^{\prime \prime}(0)}{2 !} \sigma^{2}+h^{7 / 8} \epsilon_{2}(\sigma, \tau), \\
& \tilde{a}(\sigma, \tau)^{-1}=1+h^{1 / 2} \epsilon_{3}(\sigma, \tau), \\
& \tilde{a}(\sigma, \tau)^{-2}=1+h^{1 / 2} \epsilon_{4}(\sigma, \tau), \\
& \partial_{\sigma}\left\{\tilde{a}(\sigma, \tau)^{-1}\right\}=-h^{5 / 8} \epsilon_{5}(\sigma, \tau),
\end{aligned}
$$

where, for $0<h<\frac{1}{2}, 0 \leq \tau \leq \mathcal{O}\left(h^{-\rho}\right)$ and $\sigma=\mathcal{O}\left(h^{-1 / 8}\right)$, the functions $\epsilon_{j}, j=1, \cdots, 5$, satisfy,

$$
\left|\epsilon_{1}(\sigma)\right| \leq C|\sigma|^{2}, \quad\left|\epsilon_{2}(\sigma, \tau)\right| \leq C\left(|\sigma|^{3}+h^{1 / 8} \tau\right), \quad\left|\epsilon_{3}(\sigma, \tau)\right|+\left|\epsilon_{4}(\sigma, \tau)\right|+\left|\epsilon_{5}(\sigma, \tau)\right| \leq C \tau .
$$


This gives us the following identities

$$
\begin{aligned}
-\tilde{a}^{-1} \partial_{\tau}\left(\tilde{a} \partial_{\tau}\right) & =-\partial_{\tau}^{2}+h^{1 / 2} \kappa(0) \partial_{\tau}+h^{3 / 4} \frac{\kappa^{\prime \prime}(0)}{2 !} \sigma^{2} \partial_{\tau}+h^{7 / 8} q_{1}(\sigma, \tau) \partial_{\tau}, \\
-h^{3 / 4} \tilde{a}^{-1} \partial_{\sigma}\left(\tilde{a}^{-1} \partial_{\sigma}\right) & =-h^{3 / 4} \partial_{\sigma}^{2}+h^{7 / 8}\left(h^{3 / 8} q_{2}(\sigma, \tau) \partial_{\sigma}^{2}+h^{1 / 2} q_{3}(\sigma, \tau) \partial_{\sigma}\right) .
\end{aligned}
$$

where the functions $q_{1}, q_{2}, q_{3}$ satisfy for $0<h<\frac{1}{2}, 0 \leq \tau \leq \mathcal{O}\left(h^{-\rho}\right)$ and $|\sigma|=\mathcal{O}\left(h^{-1 / 8}\right)$,

$$
\left|q_{1}(\sigma, \tau)\right| \leq C\left(|\sigma|^{3}+h^{1 / 8} \tau\right) \text { and }\left|q_{2}(\sigma, \tau)\right|+\left|q_{3}(\sigma, \tau)\right| \leq C \tau .
$$

We note for further use the expressions of the functions $q_{1}, q_{2}, q_{3}$ :

$$
\begin{aligned}
& q_{1}(\sigma, \tau)=h^{-7 / 8}\left\{-\widetilde{a}(\sigma, \tau)^{-1} \partial_{\tau}(\widetilde{a}(\sigma, \tau))-h^{1 / 2} \kappa(0)-h^{3 / 4} \frac{\kappa^{\prime \prime}(0)}{2} \sigma^{2}\right\}, \\
& q_{2}(\sigma, \tau)=h^{-1 / 8}(1-\widetilde{a}(\sigma, \tau)), \quad q_{3}(\sigma, \tau)=-\widetilde{a}(\sigma, \tau) \tau \kappa^{\prime}\left(h^{1 / 8} \sigma\right) .
\end{aligned}
$$

Consequently, we have the formal expansion of the operator $\mathcal{L}_{h}$,

$$
h^{-1} \mathcal{L}_{h}=P_{0}+h^{1 / 2} P_{2}+h^{3 / 4} P_{3}+h^{7 / 8} Q_{h},
$$

where

$$
\begin{aligned}
P_{0} & =-\partial_{\tau}^{2} \\
P_{2} & =\kappa(0) \partial_{\tau}=\kappa_{\max } \partial_{\tau}, \\
P_{3} & =-\partial_{\sigma}^{2}+\frac{\kappa^{\prime \prime}(0)}{2} \sigma^{2} \partial_{\tau}, \\
Q_{h} & =q_{1}(\sigma, \tau) \partial_{\tau}+h^{3 / 8} q_{2}(\sigma, \tau) \partial_{\sigma}^{2}+h^{3 / 8} q_{3}(\sigma, \tau) \partial_{\sigma} .
\end{aligned}
$$

Since $\kappa(0)$ is the non-degenerate maximum of $\kappa$, then $\kappa^{\prime \prime}(0)<0$. The eigenvalues of the harmonic oscillator

$$
-\partial_{\sigma}^{2}+\frac{-\kappa^{\prime \prime}(0)}{2} \sigma^{2} \quad \text { in } L^{2}(\mathbb{R})
$$

are $(2 n-1) \sqrt{\frac{-\kappa^{\prime \prime}(0)}{2}}$ with $n \in \mathbb{N}$.

The corresponding normalized eigenfunctions are denoted by $f_{n}(\sigma)$. They have the form

$$
f_{n}(\sigma)=h_{n}(\sigma) \exp \left(-\sqrt{-\frac{\kappa^{\prime \prime}(0)}{2}} \frac{\sigma^{2}}{2}\right),
$$

where the $h_{n}(\sigma)$ are the rescaled Hermite polynomials.

Define the trial state $\phi_{n} \in L^{2}(\Omega)$ in $(s, t)$ coordinates as follows:

$$
\phi_{n}(s, t)=h^{-5 / 16} \chi_{1}\left(\frac{s}{|\partial \Omega|}\right) \chi_{1}\left(\frac{t}{h^{1 / 8}}\right) u_{0}\left(h^{-1 / 2} t\right) f_{n}\left(h^{-1 / 8} s\right),
$$

where

$$
u_{0}(\tau)=\sqrt{2} \exp (-\tau)
$$

The function $u_{0}$ should be interpreted as the ground state of the model operator $\mathcal{H}_{0,0}$ introduced in (4.1).

The construction of the trial state $\phi_{n}$ is based on the simple observation that the function $g(\sigma, \tau)=u_{0}(\tau) f_{n}(\sigma)$ satisfies

$$
P_{0} g=-g, \quad P_{2} g=-\kappa_{\max } g, \quad P_{3} g=(2 n-1) g,
$$

so that, in light of the expression of the operator $\mathcal{L}_{h}$ in (6.15), we expect that (6.1) holds true. The computations that we present below will show that (6.1) is indeed true.

First, notice that, the explicit expression of $\phi_{n}$ and (3.5) give us

$$
\left\|\phi_{n}\right\|_{L^{2}(\Omega)}=1+\mathcal{O}\left(h^{3 / 4}\right) \text {. }
$$


Next, we observe that, after a change of variables, (6.15) yields (here $k_{2}=-\kappa^{\prime \prime}(0)$ )

$$
h^{-1} \mathcal{L}_{h} \phi_{n}(s, t)=\left(-1-\kappa_{\max } h^{1 / 2}+(2 n+1) \frac{k_{2}}{2} h^{3 / 4}\right) \varphi_{n}(\sigma, \tau)+R \varphi_{n}(\sigma, \tau),
$$

where $\varphi_{n}(\sigma, \tau)=\phi_{n}\left(h^{1 / 8} \sigma, h^{1 / 2} \tau\right)$ and

$$
\begin{aligned}
h^{5 / 16} R \varphi_{n}(\sigma, \tau)= & -\kappa_{\max } h^{1 / 2} h^{3 / 8} \chi_{1}^{\prime}\left(h^{3 / 8} \tau\right) \chi_{1}\left(\frac{h^{1 / 8} \sigma}{|\partial \Omega|}\right) u_{0}(\tau) f_{n}(\sigma) \\
& -h^{3 / 8}\left(2 \chi_{1}^{\prime}\left(h^{3 / 8} \tau\right)+h^{3 / 8} \chi_{1}^{\prime \prime}\left(h^{3 / 8} \tau\right)\right) \chi_{1}\left(\frac{h^{1 / 8} \sigma}{|\partial \Omega|}\right) u_{0}(\tau) f_{n}(\sigma) \\
& -h^{7 / 8}\left(2 \chi_{1}^{\prime}\left(\frac{h^{1 / 8} \sigma}{|\partial \Omega|}\right)+h^{1 / 8} \chi_{1}^{\prime \prime}\left(\frac{h^{1 / 8} \sigma}{|\partial \Omega|}\right)\right) \chi_{1}\left(h^{3 / 8} \tau\right) u_{0}(\tau) f_{n}(\sigma) \\
& +h^{7 / 8} Q_{h}\left(\chi_{1}\left(h^{3 / 8} \tau\right) \chi_{1}\left(\frac{h^{1 / 8} \sigma}{|\partial \Omega|}\right) u_{0}(\tau) f_{n}(\sigma)\right) .
\end{aligned}
$$

Using the expression of $Q_{h}$ and the exponential decay of $u_{0}$ and $f_{n}$ at infinity, it is easy to check that

$$
\int\left|R \varphi_{n}(\sigma, \tau)\right|^{2} \widetilde{a}(\sigma, \tau) h^{5 / 8} d \sigma d \tau \leq C h^{7 / 4}
$$

Now, we infer from (6.21),

$$
\begin{aligned}
\| \mathcal{L}_{h} \phi_{n}-\left(-h-\kappa_{\max } h^{3 / 2}+(2 n+1) \frac{k_{2}}{2} h^{7 / 4}\right) \phi_{n} & \|_{L^{2}(\Omega)} \\
& =h\left\|R \varphi_{n}(\sigma, \tau)\right\|_{L^{2}\left(h^{5 / 8} \widetilde{a}(\sigma, \tau) d \sigma d \tau\right)} \leq C h^{15 / 8},
\end{aligned}
$$

thereby proving (6.1).

Next, we indicate how (6.1) is useful to obtain upper bounds of the eigenvalues of the operator $\mathcal{L}_{h}$. As a consequence of the spectral theorem, (6.1) yields that, for every fixed $n \in \mathbb{N}$, there exists an eigenvalue $\widetilde{\mu}_{n}$ of the operator $\mathcal{L}_{h}$ such that

$$
\widetilde{\mu}_{n}=-h-\kappa_{\max } h^{3 / 2}+(2 n-1) \sqrt{\frac{k_{2}}{2}} h^{7 / 4}+\mathcal{O}\left(h^{15 / 8}\right) .
$$

Thus, for every $n \in \mathbb{N}$ and $h>0$ sufficiently small, we get an increasing sequence of distinct eigenvalues $\left(\widetilde{\mu}_{j}\right)_{j=1}^{n}$ of the operator $\mathcal{L}_{h}$. Clearly, the min-max eigenvalues $\left(\mu_{j}\left(\mathcal{L}_{h}\right)\right)$ of the operator $\mathcal{L}_{h}$ will satisfy

$$
\forall j \in\{1,2, \cdots, n\}, \quad \mu_{j}\left(\mathcal{L}_{h}\right) \leq \max _{1 \leq i \leq j} \widetilde{\mu}_{i} \leq \widetilde{\mu}_{j} .
$$

In that way, we arrive at:

Theorem 6.1. Let $n \in \mathbb{N}$. There exist constants $h_{n} \in(0,1)$ and $C_{n}>0$ such that the min-max eigenvalues $\left(\mu_{j}\left(\mathcal{L}_{h}\right)\right)_{j=1}^{n}$ of the operator $\mathcal{L}_{h}$ satisfy, for all $h \in\left(0, h_{n}\right)$,

$$
\mu_{j}\left(\mathcal{L}_{h}\right) \leq-h-\kappa_{\max } h^{3 / 2}+(2 j-1) \sqrt{\frac{-\kappa^{\prime \prime}(0)}{2}} h^{7 / 4}+r_{j}(h)
$$

where

$$
\left|r_{j}(h)\right| \leq C_{n} h^{15 / 8}
$$




\section{REFINED TRIAL STATES}

\subsection{Main Result.}

In this section, we construct trial states that will give us, together with the lower bounds that we will derive in the next section, an accurate expansion of the eigenvalues of the operator $\mathcal{L}_{h}$ valid to any order.

In (6.15), we derived the expression of the operator $\mathcal{L}_{h}$ in the re-scaled boundary coordinates $(\sigma, \tau)=\left(h^{-1 / 8} s, h^{-1 / 2} t\right)$. The expansion in (6.15) involves the operators $P_{0}, P_{2}, P_{3}, Q_{h}$ in (6.16). The key to the construction of the trial states is to expand the operator $Q_{h}$ in powers of $h^{1 / 8}$.

The functions $q_{1}, q_{2}$ and $q_{3}$ in (6.16) admit Taylor expansions that can be rearranged in the form:

$$
\begin{aligned}
& q_{1}(\sigma, \tau)=\sum_{j=0}^{\infty} q_{1, j}(\sigma, \tau) h^{j / 8}, \\
& h^{3 / 8} q_{2}(\sigma, \tau)=\sum_{j=0}^{\infty} q_{2, j}(\sigma, \tau) h^{j / 8}, \\
& h^{3 / 8} q_{3}(\sigma, \tau)=\sum_{j=0}^{\infty} q_{3, j}(\sigma, \tau) h^{j / 8},
\end{aligned}
$$

with the functions $q_{k, j}(\sigma, \tau)$ being polynomial functions of $(\sigma, \tau)$. The operator $Q_{h}$ in (6.16) admits the formal expansion

$$
Q_{h}=\sum_{j=0}^{\infty} h^{j / 8} Q_{j}
$$

where

$$
Q_{j}=q_{1, j}(\sigma, \tau) \partial_{\tau}+q_{2, j}(\sigma, \tau) \partial_{\sigma}^{2}+q_{3, j}(\sigma, \tau) \partial_{\sigma}
$$

Furthermore, for every $p \geq 1, j \geq 0$ and $f \in \mathcal{S}\left(\mathbb{R} \times \overline{\mathbb{R}_{+}}\right), \quad Q_{j} f \in L^{p}\left(\mathbb{R} \times \mathbb{R}_{+}\right)$.

Remember from (6.19) that $u_{0}(\tau)=\sqrt{2} \exp (-\tau)$ and $f_{n}(\sigma)$ is the $n$-th normalized eigenfunction of the harmonic oscillator $H_{\text {harm }}=-\partial_{\sigma}^{2}+\frac{k_{2}}{2} \sigma^{2}$.

For all $M \geq 0$, we introduce the two operators:

$$
\widetilde{\mathcal{L}}_{h, M}=P_{0}+h^{1 / 2} P_{2}+h^{3 / 4} P_{3}+h^{7 / 8} F_{M} \quad \text { and } \quad F_{M}=\sum_{j=0}^{M} h^{j / 8} Q_{j} .
$$

Thanks to (6.15) and (7.2), for every $f \in \mathcal{S}\left(\mathbb{R} \times \mathbb{R}_{+}\right)$, there exists a constant $C>0$ such that, for all $h \in(0,1)$,

$$
\left\|h^{-1} \mathcal{L}_{h} f-\widetilde{\mathcal{L}}_{h, M} f\right\|_{L^{2}\left(\mathbb{R} \times \mathbb{R}_{+}\right)} \leq C h^{\frac{7}{8}+\frac{M+1}{8}} .
$$

Let $n \in \mathbb{N}$. We will construct a sequence of real numbers $\left(\zeta_{j, n}\right)_{j=0}^{\infty}$ and two sequences of real-valued Schwartz functions $\left(v_{n, j}\right)_{j=1}^{\infty} \subset \mathcal{S}(\mathbb{R}),\left(g_{n, j}\right)_{j=0}^{\infty} \subset \mathcal{S}\left(\mathbb{R} \times \overline{\mathbb{R}_{+}}\right)$such that

$$
\forall j,\left.\quad \partial_{\tau} g_{n, j}\right|_{\tau=0}=-\left.g_{n, j}\right|_{\tau=0},
$$

and for all $M \in \mathbb{N}$, the function

$$
\Psi_{n, M}(\sigma, \tau)=u_{0}(\tau) f_{n}(\sigma)+\sum_{j=1}^{M+1} h^{j / 8} u_{0}(\tau) v_{n, j}(\sigma)+h^{7 / 8} \sum_{j=0}^{M} h^{j / 8} g_{n, j}(\sigma, \tau)
$$

is in the Schwartz space $\mathcal{S}\left(\mathbb{R} \times \mathbb{R}_{+}\right)$and satisfies (for all $h \in\left(0, h_{n, M}\right)$ )

$$
\left\|\left(\widetilde{\mathcal{L}}_{h, M}-\mu_{h, M}\right) \Psi_{n, M}\right\|_{L^{2}\left(\mathbb{R} \times \mathbb{R}_{+}\right)} \leq C_{n, M} h^{\frac{7}{8}+\frac{M+1}{8}},
$$


where

$$
\mu_{n, M}=-1-h^{1 / 2} \kappa_{\max }+h^{3 / 4} \sqrt{\frac{k_{2}}{2}}(2 n-1)+h^{7 / 8} \sum_{j=0}^{M} \zeta_{j, n} h^{j / 8},
$$

$C_{n, M}>0$ and $h_{n, M}$ are two constants determined by the values of $n$ and $M$ solely.

Let us mention that, for every $j$, our definition of the function $g_{n, j}(\sigma, \tau)$ ensures that it is a finite sum of functions having the simple form $F(\sigma) \times U(\tau)$.

Before we proceed in the construction of the aforementioned sequences, we show how they give us refined expansions of the eigenvalues of the operator $\mathcal{L}_{h}$. Let $\chi_{1}$ be the cut-off function as in (6.18). Define a function $\phi_{n, M}$ in $L^{2}(\Omega)$ by means of the boundary coordinates as follows:

$$
\phi_{n, M}(s, t)=\chi_{1}\left(\frac{s}{|\partial \Omega|}\right) \chi_{1}\left(\frac{t}{h^{1 / 8}}\right) \Psi_{n, M}\left(h^{-1 / 8} s, h^{-1 / 2} t\right) .
$$

The function $\phi_{n, M}$ is in the domain of the operator $\mathcal{L}_{h}$ because the functions $g_{n, j}$ and $u_{0}$ satisfy the Robin condition at $\tau=0$. Since all involved functions in the expression of $\Psi_{n, M}$ are in the Schwartz space, multiplication by cut-off functions will produce small errors in the various calculations-all the error terms are $\mathcal{O}\left(h^{\infty}\right)$. Also, since the function $u_{0}(\tau) f_{n}(\sigma)$ is normalized in $L^{2}\left(\mathbb{R} \times \mathbb{R}_{+}\right)$, the norm of $\phi_{n, M}$ in $L^{2}(\Omega)$ is equal to $1+o(1)$, as $h \rightarrow 0_{+}$.

Thanks to (7.5) and (7.6), we get that

$$
\left\|\mathcal{L}_{h} \phi_{n, M}-h \mu_{n, M} \phi_{n, M}\right\|_{L^{2}(\Omega)}=\mathcal{O}\left(h^{\frac{7}{8}+\frac{M+1}{8}}\right)\left\|\Psi_{n, M}\right\|_{L^{2}(\Omega)} .
$$

The spectral theorem now gives us:

Theorem 7.1. Let $n \in \mathbb{N}$. For every $M \geq 0$, there exists an eigenvalue $\widetilde{\mu}_{n}(h)$ of the operator $\mathcal{L}_{h}$ such that, as $h \rightarrow 0_{+}$,

$$
\tilde{\mu}_{n}(h)=-h-h^{3 / 2} \kappa_{\max }+h^{3 / 4} \sqrt{\frac{k_{2}}{2}}(2 n-1)+h^{7 / 8} \sum_{j=0}^{M} \zeta_{j, n} h^{j / 8}+\mathcal{O}\left(h^{\frac{7}{8}+\frac{M+1}{8}}\right) .
$$

The rest of the section is devoted to the construction of the numbers $\zeta_{j, n}$ and the functions $\Psi_{n, M}$. That will be done by an iteration process. For simplicity, we will drop $n$ from the notation and write $\zeta_{j}, \Psi_{j}, \cdots$.

\subsection{The sequence $\left(\zeta_{j, n}\right)$.}

Construction of $\left(\zeta_{n, 0}, \Psi_{n, 0}\right)=\left(\zeta_{0}, \Psi_{0}\right)$.

We want to find a real number $\zeta_{0}$ and two functions $v_{1}(\sigma), g_{0}(\sigma, \tau)$ such that

$$
\Psi_{0}(\sigma, \tau)=u_{0}(\tau) f_{n}(\sigma)+h^{1 / 8} u_{0}(\tau) v_{1}(\sigma)+h^{7 / 8} g_{0}(\sigma, \tau)
$$

and

$$
\mu_{0}=-1-h^{1 / 2} \kappa_{\max }+h^{3 / 4} \sqrt{\frac{k_{2}}{2}}(2 n-1)+\zeta_{0} h^{7 / 4}
$$

satisfy (7.5) for $M=0$.

Recall that the expression of the operator $\widetilde{\mathcal{L}}_{h, M}$ for $M=0$ is

$$
\widetilde{\mathcal{L}}_{h, 0}=P_{0}+h^{1 / 2} P_{2}+h^{3 / 4} P_{3}+h^{7 / 8} F_{0} .
$$

For every Schwartz function $v(\sigma)$, we have the two simple identities,

$$
\left(P_{0}+h^{1 / 2} P_{2}\right) u_{0}(\tau) v(\sigma)=\left(-1-h^{1 / 2} \kappa_{\max }\right) u_{0}(\tau) v(\sigma) \quad \text { and } \quad P_{3} u_{0}(\tau) v(\sigma)=u_{0}(\tau) H_{\mathrm{harm}} v(\sigma),
$$


where $H_{\text {harm }}=-\frac{d^{2}}{d \sigma^{2}}+\frac{k_{2}}{2} \sigma^{2}$. A straightforward computation yields

$$
\begin{aligned}
& \left(\widetilde{\mathcal{L}}_{h, 0}-\mu_{0}\right) \Psi_{0} \\
& =h^{7 / 8}\left[\left(P_{0}+1\right) g_{0}(\sigma, \tau)+u_{0}(\tau)\left(H_{\text {harm }}-\sqrt{\frac{k_{2}}{2}}(2 n-1)\right) v_{1}(\sigma)+\left(F_{0}-\zeta_{0}\right) u_{0}(\tau) f_{n}(\sigma)\right] \\
& +h^{7 / 8}\left[\left(h^{1 / 2} P_{2}+h^{3 / 4} P_{3}+h^{7 / 8} F_{0}+h^{1 / 2} \kappa_{\max }-h^{3 / 4} \sqrt{\frac{k_{2}}{2}}(2 n-1)-\zeta_{0} h^{7 / 4}\right) g_{0}(\sigma, \tau)\right] \\
& +h\left[F_{0}-\zeta_{0}\right] u_{0}(\tau) v_{1}(\sigma) .
\end{aligned}
$$

In order that (7.5) is satisfied for $M=0$, it is sufficient to select $\zeta_{0}, g_{0}(\sigma, \tau)$ and $v_{1}(\sigma)$ as follows

$$
\left(P_{0}+1\right) g_{0}(\sigma, \tau)+u_{0}(\tau)\left(H_{\mathrm{harm}}-\sqrt{\frac{k_{2}}{2}}(2 n-1)\right) v_{1}(\sigma)+\left(F_{0}-\zeta_{0}\right) u_{0}(\tau) f_{n}(\sigma)=0 .
$$

We can select $\zeta_{0}$ such that

$$
\left(F_{0}-\zeta_{0}\right) u_{0}(\tau) f_{n}(\varsigma) \perp u_{0}(\tau) f_{n}(\sigma) \text { in } L^{2}\left(\mathbb{R} \times \mathbb{R}_{+}\right) .
$$

This is given by the formula:

$$
\zeta_{0}=\left\langle F_{0}\left(u_{0} \otimes f_{n}\right), u_{0} \otimes f_{n}\right\rangle_{L^{2}\left(\mathbb{R} \times \mathbb{R}_{+}\right)} .
$$

Consequently,

$$
h_{1}(\sigma):=-\int_{0}^{\infty} u_{0}(\tau)\left(F_{0}-\zeta_{0}\right) u_{0}(\tau) f_{n}(\sigma) d \tau \perp f_{n}(\sigma) \quad \text { in } L^{2}(\mathbb{R}) .
$$

Since $H_{\text {harm }}-\sqrt{\frac{k_{2}}{2}}(2 n-1)$ can be inverted in the orthogonal complement of the $n$-th eigenfunction $f_{n}(\sigma)$, we may select $v_{1}(\sigma)$ to be

$$
v_{1}(\sigma)=\left(H_{\text {harm }}-\sqrt{\frac{k_{2}}{2}}(2 n-1)\right)^{-1}\left(h_{1}(\sigma)\right) .
$$

As a consequence of the choice of $\zeta_{0}$ and $v_{1}(\sigma)$, we get that, for all $\sigma$,

$$
\begin{aligned}
w_{1}(\sigma, \cdot):=-u_{0}(\cdot)\left(H_{\mathrm{harm}}-\sqrt{\frac{k_{2}}{2}}(2 n-1)\right) v_{1}(\sigma)-\left(F_{0}-\zeta_{0}\right) u_{0}(\cdot) f_{n}(\sigma) & \\
& =-u_{0}(\cdot) h_{1}(\sigma)-\left(F_{0}-\zeta_{0}\right) u_{0}(\cdot) f_{n}(\sigma)
\end{aligned}
$$

is in the orthogonal complement of $\left\{u_{0}(\tau)\right\}$ in $L^{2}\left(\mathbb{R}_{+}\right)$, i.e.

$$
\int_{0}^{\infty} w_{1}(\sigma, \tau) u_{0}(\tau) d \tau=0
$$

Furthermore, since $F_{0}=q_{1,0} \partial_{\tau}+q_{2,0} \partial_{\sigma}^{2}+q_{3,0} \partial_{\sigma}$ and every $q_{i, 0}$ is a polynomial in $(\sigma, \tau)$, then the function $w_{1}(\sigma, \tau)$ can be expressed in the form

$$
w_{1}(\sigma, \tau)=-h_{1}(\sigma) u_{0}(\tau)+G_{1}(\sigma) u_{1}(\tau)+G_{2}(\sigma) u_{2}(\tau)+G_{3}(\sigma) u_{3}(\tau)
$$

where the functions $h_{1}, u_{i}$ and $G_{i}$ are Schwartz functions (they inherit this property from the functions $u_{0}$ and $f_{n}$ ).

The operator $P_{0}+1$ can be inverted in the orthogonal complement of $\left\{u_{0}(\tau)\right\}$, and the inverse is an operator in $L^{2}\left(\mathbb{R}_{+}\right)$which sends $\mathcal{S}\left(\overline{\mathbb{R}_{+}}\right)$into itself. The proof of this is standard and follows the same argument as in [5, Lemma A.5]. In that way, we may select $g_{0}(\sigma, \tau)$ to be

$$
g_{0}(\sigma, \cdot)=\left(P_{0}+1\right)^{-1}\left(w_{1}(\sigma, \cdot)\right),
$$

and this function is in the Schwarz space $\mathcal{S}\left(\mathbb{R} \times \overline{\mathbb{R}}_{+}\right)$, thanks to the decomposition of $w_{1}$ in (7.12). 
Now, for $M=1$, the estimate in (7.6) is verified. Furthermore, returning to (7.9) we have proved that:

$$
\left(\widetilde{\mathcal{L}}_{h, 0}-\mu_{0}\right) \Psi_{0}=h \sum_{j=0}^{10} h^{j / 8} r_{0, j}(\sigma, \tau),
$$

where $r_{0, j}(\sigma, \tau)$ are Schwartz functions.

The iteration process.

Here we suppose that we have selected $\left(\zeta_{0}, \cdots, \zeta_{M} ; \Psi_{0}, \cdots \Psi_{M}\right)$ such that (7.5) and the following induction hypothesis are satisfied:

$$
\left(\widetilde{\mathcal{L}}_{h, M}-\mu_{M}\right) \Psi_{M}=h^{\frac{7}{8}+\frac{M+1}{8}} \sum_{j=0}^{N_{M}} h^{j / 8} r_{M, j}(\sigma, \tau),
$$

where $r_{M, j}(\sigma, \tau)$ are Schwartz functions. Thanks to (7.13), the condition in (7.14) holds for $M=0$. We have to select $\zeta_{M+1}$ and two functions $v_{M+2}(\sigma), g_{M+1}(\sigma, \tau)$ such that,

$$
\Psi_{M+1}=\Psi_{M}+h^{\frac{M+2}{8}} u_{0}(\tau) v_{M+1}(\sigma)+h^{\frac{7}{8}+\frac{M+1}{8}} g_{M+1}(\sigma, \tau) \quad \text { and } \quad \mu_{M+1}=\mu_{M}+\zeta_{M+1} h^{\frac{7}{8}+\frac{M+1}{8}}
$$

satisfy (7.5) and (7.14) for $M$ replaced by $M+1$ (and some collection of Schwartz functions $\left.r_{M+1, j}(\sigma, \tau)\right)$.

Notice that we have

$$
\widetilde{\mathcal{L}}_{h, M+1}=\widetilde{\mathcal{L}}_{h, M}+h^{\frac{7}{8}+\frac{M+1}{8}} Q_{M+1},
$$

and

$$
\begin{aligned}
& \left(\widetilde{\mathcal{L}}_{h, M+1}-\mu_{M+1}\right) \Psi_{M+1} \\
& =\left(\widetilde{\mathcal{L}}_{h, M}-\mu_{M}\right) \Psi_{M} \\
& +h^{\frac{7}{8}+\frac{M+1}{8}}\left[\left(P_{0}+1\right) g_{M+1}(\sigma, \tau)+u_{0}(\tau)\left(H_{\mathrm{harm}}-\sqrt{\frac{k_{2}}{2}}(2 n-1)\right) v_{M+2}(\sigma)+\left(Q_{M+1}-\zeta_{M+1}\right) u_{0}(\tau) f_{n}(\sigma)\right] \\
& +h^{\frac{7}{8}+\frac{M+1}{8}}\left[\left(Q_{M+1}-\zeta_{M+1}\right)\left(\Psi_{M}-u_{0}(\tau) f_{n}(\sigma)\right)\right] \\
& +h^{\frac{7}{8}+\frac{M+1}{8}}\left[\left(h^{1 / 2} P_{2}+h^{3 / 4} P_{3}+h^{7 / 8} F_{M+1}-\left(\mu_{M}+1\right)\right) g_{M+1}(\sigma, \tau)\right] \\
& +h^{\frac{7}{8}+\frac{M+1}{8}}\left[h^{1 / 8} F_{M+1}-\left(\mu_{M+1}+1+h^{1 / 2} \kappa_{\max }-h^{3 / 4} \sqrt{\frac{k_{2}}{2}}(2 n-1)\right)\right] u_{0}(\tau) v_{M+2}(\sigma) .
\end{aligned}
$$

It is sufficient to select $\left(\zeta_{M+1}, v_{M+2}, g_{M+1}\right)$ such that

$$
\begin{aligned}
\left(P_{0}+1\right) g_{M+1}(\sigma, \tau)+u_{0}(\tau)\left(H_{\text {harm }}-\sqrt{\frac{k_{2}}{2}}(2 n-1)\right) v_{M+2}(\sigma) & \\
& +\left(Q_{M+1}-\zeta_{M+1}\right) u_{0}(\tau) f_{n}(\sigma)+r_{M, 0}(\sigma, \tau)=0 .
\end{aligned}
$$

We select $\zeta_{M+1}$ such that

$$
\left(Q_{M+1}-\zeta_{M+1}\right) u_{0}(\tau) f_{n}(\sigma)+r_{M, 0}(\sigma, \tau) \perp u_{0}(\tau) f_{n}(\sigma) \text { in } L^{2}\left(\mathbb{R} \times \mathbb{R}_{+}\right),
$$

i.e.

$$
\zeta_{M+1}=\left\langle Q_{M+1}\left(u_{0} \otimes f_{n}\right), u_{0} \otimes f_{n}\right\rangle_{L^{2}\left(\mathbb{R} \times \mathbb{R}_{+}\right)}+\left\langle r_{M, 0}, u_{0} \otimes f_{n}\right\rangle_{L^{2}\left(\mathbb{R} \times \mathbb{R}_{+}\right)} .
$$

Consequently,

$$
h_{M+2}(\sigma):=-\int_{0}^{\infty} u_{0}(\tau)\left(\left(Q_{M+1}-\zeta_{M+1}\right) u_{0}(\tau) f_{n}(\sigma)+r_{M, 0}(\sigma, \tau)\right) d \tau \perp f_{n}(\sigma) \quad \text { in } L^{2}(\mathbb{R}) .
$$

We select $v_{M+2}(\sigma)$ as follows

$$
v_{M+2}(\sigma)=\left(H_{\text {harm }}-\sqrt{\frac{k_{2}}{2}}(2 n-1)\right)^{-1}\left(h_{M+2}(\sigma)\right) .
$$


As a consequence of the choice of $\zeta_{M+1}$ and $v_{M+2}(\sigma)$, we get that, for all $\sigma$,

$w_{M+2}(\sigma, \cdot):=-u_{0}(\cdot)\left(H_{\mathrm{harm}}-\sqrt{\frac{k_{2}}{2}}(2 n-1)\right) v_{M+2}(\sigma)-\left(Q_{M+1}-\zeta_{M+1}\right) u_{0}(\tau) f_{n}(\sigma)-r_{M, 0}(\sigma, \tau) \perp u_{0}(\cdot)$ in $L^{2}\left(\mathbb{R}_{+}\right)$.

Finally, we select $g_{M+1}(\sigma, \tau)$ as follows

$$
g_{M+1}(\sigma, \cdot)=\left(P_{0}+1\right)^{-1}\left(w_{M+2}(\sigma, \cdot)\right) .
$$

Having selected $\zeta_{M+1}, v_{M+2}$ and $g_{M+1}$, the expansions in (7.15) and (7.14) yield that

$$
\left(\widetilde{\mathcal{L}}_{h, M+1}-\mu_{M}\right) \Psi_{M+1}=h^{\frac{7}{8}+\frac{M+2}{8}} \sum_{j=0}^{N_{M+1}} h^{j / 8} r_{M+1, j}(\sigma, \tau),
$$

where $r_{M+1, j}(\sigma, \tau)$ are Schwartz functions.

7.3. Vanishing of even indexed $\zeta_{j, n}$.

Let us start by observing that $f_{1}(\sigma)$ is a Gaussian, hence an even function. The other eigenfunctions $f_{n}(\sigma)$ are generated from $f_{1}(\sigma)$ by the recursive relation

$$
f_{n}(\sigma)=c_{n}\left(L^{+}\right)^{n-1} f_{1}(\sigma),
$$

where the constant $c_{n}$ normalizes $f_{n}$ in $L^{2}(\mathbb{R})$, and $L^{+}=-\frac{d}{d \sigma}+\sigma$. The operator $L^{+}$transforms even functions to odd functions, and odd functions to even functions. In that way, we observe that, $f_{n}(\sigma)$ is even when $n$ is odd, and odd otherwise.

Next we examine carefully the terms $q_{k}(\sigma, \tau), k \in\{1,2,3\}$. Thanks to (6.14), we see that,

$$
q_{1}(\sigma, \tau)=h^{-3 / 8} \alpha\left(h^{1 / 8} \sigma, h^{1 / 2} \tau\right),
$$

with $\alpha(x, y)$ being a smooth function. We will abuse notation and identify $q_{1}$ with its Taylor expansion. In that way we write,

$$
\begin{aligned}
q_{1}(\sigma, \tau)=h^{-3 / 8} \sum_{m=3}^{\infty} \sum_{k=0}^{m} \frac{\partial^{m} \alpha}{\partial x^{k} \partial y^{m-k}}(0,0)\left(h^{1 / 8}\right)^{k}\left(h^{1 / 2}\right)^{m-k} \sigma^{k} \tau^{m-k} \\
=\sum_{m=3}^{\infty} \sum_{k=0}^{m} \frac{\partial^{m} \alpha}{\partial x^{k} \partial y^{m-k}}(0,0) h^{\frac{k-3}{8}} h^{\frac{4(m-k)}{8}} \sigma^{k} \tau^{m-k} .
\end{aligned}
$$

Consequently, in the above expansion of $q_{1}(\sigma, \tau)$, we see that the coefficients of the terms with odd powers of $\sigma$ have even powers of $h^{1 / 8}$ (actually $k$ is odd iff $(k-3)+4(m-k)$ is even). Returning to the expansion of $q_{1}(\sigma, \tau)$ in (7.1), we get that, for all even $j$, the function $q_{1, j}(\sigma, \tau)$ is an odd function of $\sigma$, while for odd $j$, it is an even function of $\sigma$.

A similar analysis of the terms $q_{2}(\sigma, \tau)$ and $q_{3}(\sigma, \tau)$ shows that, for all $j$, the term $q_{2, j}(\sigma, \tau)$ is an odd function of $\sigma$ iff $j$ is even, and $q_{3, j}(\sigma, \tau)$ is an odd function of $\sigma$ iff $j$ is odd.

By the discussion on the parity of the function $f_{n}(\sigma)$, we see that, if $M$ is even,

$$
Q_{M} u_{0}(\tau) f_{n}(\sigma)=u_{0}(\tau)\left(-q_{1, M} f_{n}(\sigma)+q_{2, M} f_{n}^{\prime \prime}(\sigma)+q_{3, j} f_{n}^{\prime}(\sigma)\right)
$$

is an odd function of $\sigma$ when $n$ is odd, and it is an even function when $n$ is even. Consequently, for every $n$, if $M$ is even, then the function

$$
\left(Q_{M} u_{0}(\tau) f_{n}(\sigma)\right) u_{0}(\tau) f_{n}(\sigma)
$$

is an odd function of $\sigma$.

Consequently, for all values of $\tau$,

$$
\int_{\mathbb{R}}\left(Q_{M} u_{0}(\tau) f_{n}(\sigma)\right) u_{0}(\tau) f_{n}(\sigma) d \sigma=0
$$


Similarly, one can follow by recursion the parity of $r_{M, 0}$ with respect to $\sigma$ and get for $M$ even

$$
\int_{\mathbb{R}} r_{M, 0}(\sigma, \tau) u_{0}(\tau) f_{n}(\sigma) d \sigma=0 .
$$

Inserting this into (7.11) and (7.16), we get that $\zeta_{M, n}=0$ for every odd $M$.

\section{LOWER BOUNDS FOR THE LOW-LYING EIGENVALUES}

8.1. Useful operators. Let $\rho \in(0,1)$. The number $\rho$ will be selected as $\rho=\frac{1}{8}+\eta$ with $\eta \in\left(0, \frac{1}{24}\right)$. In the sequel, $\sigma$ and $\tau$ will denoted two variables. The variable $\sigma$ will live in $\mathbb{R}$ (or in the interval $\left(h^{-\eta}, h^{\eta}\right)$. The variable $\tau$ will live in the interval $\left.\left(0, h^{-\rho}\right)\right)$. When writing $\mathbb{R}_{\sigma}$, we mean that that the set of real numbers $\mathbb{R}$ is the space where the variable $\sigma$ varies.

We introduce the following operators in $\mathcal{L}\left(L^{2}\left(\mathbb{R}_{\sigma}\right), L^{2}\left(\mathbb{R}_{\sigma} \times\left(0, h^{-\rho}\right)\right)\right)$, and in $\mathcal{L}\left(L^{2}\left(\mathbb{R}_{\sigma} \times\left(0, h^{-\rho}\right)\right), L^{2}\left(\mathbb{R}_{\sigma}\right)\right)$ respectively.

The $L^{2}$-spaces that appear are equipped with the standard $L^{2}$-norm. Recall the normalized eigenfunction $u_{0, h}$ introduced in Remark 4.3 Define the bounded operators $R_{0}^{+}$and $R_{0}^{-}$as follows:

$$
\begin{aligned}
& \left(R_{0}^{+} v\right)(\sigma, \tau)=u_{0, h}(\tau) v(\sigma), \\
& \left(R_{0}^{-} v\right)(\sigma)=\int_{0}^{h^{-\rho}} u_{0, h}(\tau) v(\sigma, \tau) d \tau .
\end{aligned}
$$

Note that $R_{0}^{+} R_{0}^{-}$is the orthogonal projection, in $L^{2}\left(\mathbb{R} \times\left(0, h^{-\rho}\right)\right)$, on the space $L^{2}\left(\mathbb{R}_{\sigma}\right) \otimes\left\{\mathbb{R} u_{0, h}\right\}$. The operator norms of $R_{0}^{+}$and $R_{0}^{-}$are both equal to 1 . The function $R_{0}^{+} v \in L^{2}\left(\mathbb{R}_{\sigma} \times\left(0, h^{-\rho}\right)\right)$ will be extended by zero to a function in $L^{2}\left(\mathbb{R}^{2}\right)$.

In the subsequent subsections, we will need two operators that approximate $R_{0}^{-}$. Define the two operators $R_{0, h}^{-}$and $\widetilde{R}_{0, h}^{-}$in $\mathcal{L}\left(L^{2}\left(\left(-h^{-\eta}, h^{-\eta}\right) \times\left(0, h^{-\rho}\right)\right) ; L^{2}\left(\left(-h^{-\eta}, h^{-\eta}\right)\right)\right)$ as follows:

$$
\begin{aligned}
& R_{0, h}^{-} v=\int_{0}^{h^{-\rho}} u_{0, h}(\tau) v(\sigma, \tau) \widetilde{a}(\sigma, \tau) d \tau, \\
& \widetilde{R}_{0, h}^{-} v=\int_{0}^{h^{-\rho}} u_{0, h}(\tau) v(\sigma, \tau) \widetilde{a}(\sigma, \tau)^{-1} d \tau,
\end{aligned}
$$

where $\widetilde{a}$ is introduced in (6.4).

The operators $R_{0, h}^{-}$and $\widetilde{R}_{0, h}^{-}$are approximations of $R_{0}^{-}$relative to the operator norm in $\mathcal{L}\left(L^{2}\left(\left(-h^{-\eta}, h^{-\eta}\right) \times\left(0, h^{-\rho}\right)\right) ; L^{2}\left(\left(-h^{-\eta}, h^{-\eta}\right)\right)\right.$ since, by (6.5), (6.7) and (6.10),

$$
\left\|R_{0, h}^{-}-R_{0}^{-}\right\| \leq C h^{\frac{1}{2}-\rho} \quad \text { and } \quad\left\|\widetilde{R}_{0, h}^{-}-R_{0}^{-}\right\| \leq C h^{\frac{1}{2}-\rho} .
$$

\subsection{Approximate eigenfunctions for the harmonic oscillator.}

\subsubsection{Construction of the trial states and preliminary estimates.}

We use the eigenfunctions of the Laplacian $\mathcal{L}_{h}$ to construct approximate eigenfunctions of the harmonic oscillator

$$
H_{\text {harm }}=-\frac{d^{2}}{d \sigma^{2}}+\frac{k_{2}}{2} \sigma^{2} \quad \text { in } L^{2}(\mathbb{R}) .
$$

Let $n \in \mathbb{N}, \eta \in(0,1 / 8)$, and $v_{h, n}$ be a normalized eigenfunction of $\mathcal{L}_{h}$ associated with the $n$-th eigenvalue $\mu_{n}(h)$.

Recall from Theorem 6.1 that $\mu_{n}(h)$ satisfies

$$
\mu_{n}(h) \leq-h-\kappa_{\max } h^{3 / 2}+M_{n} h^{7 / 4},
$$


where $M_{n}>0$ is a constant. Define the functions $\psi_{h, n}$ and $\widetilde{\psi}_{h, n}$ as follows:

$$
\begin{aligned}
& \psi_{h, n}(s, t)=\chi\left(\frac{s}{h^{\frac{1}{8}-\eta}}\right) \chi\left(\frac{t}{h^{\frac{1}{2}-\rho}}\right) v_{h, n}(s, t), \\
& \widetilde{\psi}_{h, n}(\sigma, \tau)=h^{5 / 16} \psi_{h, n}\left(h^{1 / 8} \sigma, h^{1 / 2} \tau\right) .
\end{aligned}
$$

The function $\chi \in C_{c}^{\infty}(\mathbb{R})$ satisfies $0 \leq \chi \leq 1$ in $\mathbb{R}, \chi=1$ in $\left[-\frac{1}{2}, \frac{1}{2}\right]$ and the support of $\chi$ is in $[-1,1]$.

Notice that the function $\psi_{h, n}$ is defined in $\Omega$ by means of the boundary coordinates in (3.2).

Proposition 8.1. There holds:

(1) $\left\|\mathcal{L}_{h} \psi_{h, n}-\mu_{n}(h) \psi_{h, n}\right\|_{2}=\mathcal{O}\left(h^{\infty}\right)$;

(2) $\left\|\psi_{h, n}-v_{h, n}\right\|_{2}=\mathcal{O}\left(h^{\infty}\right)$;

(3) $\left\|\widetilde{\psi}_{h, n}-R_{0}^{+} R_{0}^{-} \widetilde{\psi}_{h, n}\right\|_{2}=o(1)$;

(4) $\left\|R_{0}^{-} \widetilde{\psi}_{h, n}\right\|_{L^{2}(\mathbb{R})}=1+o(1)$;

(5) If $i \neq j$, then the functions $R_{0}^{-} \widetilde{\psi}_{h, i}$ and $R_{0}^{-} \widetilde{\psi}_{h, j}$ are almost orthogonal in the following sense,

$$
\left\langle R_{0}^{-} \widetilde{\psi}_{h, i}, R_{0}^{-} \widetilde{\psi}_{h, j}\right\rangle_{L^{2}(\mathbb{R})}=o(1) .
$$

Proof. The assertions in (1) and (2) follow from Theorems 5.1 and 5.5.

Proof of (3):

Thanks to the assertions in (1) and (2), we may write,

$$
q_{h}\left(\psi_{h, n}\right) \leq \mu_{n}(h)+\mathcal{O}\left(h^{\infty}\right) .
$$

We express $q_{h}\left(\psi_{h, n}\right)$ in boundary coordinates to write (see (3.4l))

$$
\begin{aligned}
\int_{-|\partial \Omega| / 2}^{|\partial \Omega| / 2} \int_{0}^{h^{\frac{1}{2}-\rho}}\left|h \partial_{t} \psi_{h, n}\right|^{2}(1-t \kappa(s)) d t d s-h^{3 / 2} \int_{-|\partial \Omega| / 2}^{|\partial \Omega| / 2} \mid \psi_{h, n}(s, t & =0)\left.\right|^{2} d \sigma \\
& \leq \mu_{n}(h)+\mathcal{O}\left(h^{\infty}\right) .
\end{aligned}
$$

Using that $\kappa(\cdot)$ is bounded, $\rho \in(0,1 / 2)$ and Theorem 5.1, we get

$$
\left.\left|\int_{-|\partial \Omega| / 2}^{|\partial \Omega| / 2} \int_{0}^{h^{\frac{1}{2}-\rho}}\right| h \partial_{t} \psi_{h, n}\right|^{2} t \kappa(s) d t d s \mid \leq C h h^{\frac{1}{2}-\rho}
$$

and, as a consequence of (8.9),

$$
\int_{-|\partial \Omega| / 2}^{|\partial \Omega| / 2} \int_{0}^{h^{\frac{1}{2}-\rho}}\left|h \partial_{t} \psi_{h, n}\right|^{2} d t d s-h^{3 / 2} \int_{-|\partial \Omega| / 2}^{|\partial \Omega| / 2}\left|\psi_{h, n}(s, t=0)\right|^{2} d \sigma \leq \mu_{n}(h)+\mathcal{O}\left(h^{\frac{3}{2}-\rho}\right) .
$$

We apply the change of variables $(s, t)=\left(h^{1 / 8} \sigma, h^{1 / 2} \tau\right)$ and note that the support of the function $\widetilde{\psi}_{h, n}$ is in $\left\{(\sigma, \tau) \in\left(-h^{-\eta}, h^{\eta}\right) \times\left[0, h^{-\rho}\right)\right\}$. In that way, we obtain

$$
h\left(\int_{-h^{-\eta}}^{h^{-\eta}} \int_{0}^{h^{-\rho}}\left|\partial_{\tau} \widetilde{\psi}_{h, n}\right|^{2} d \tau d \sigma-\int_{-h^{-\eta}}^{h^{-\eta}}\left|\widetilde{\psi}_{h, n}(\sigma, \tau=0)\right|^{2} d \sigma\right) \leq \mu_{n}(h)+\mathcal{O}\left(h^{\frac{3}{2}-\rho}\right) .
$$

We have the following simple decomposition of $\widetilde{\psi}_{h, n}$,

$$
\widetilde{\psi}_{h, n}(\sigma, \tau)=R_{0}^{+} R_{0}^{-} \widetilde{\psi}_{h, n}(\sigma, \tau)+\left(\widetilde{\psi}_{h, n}-R_{0}^{+} R_{0}^{-} \widetilde{\psi}_{h, n}\right)(\sigma, \tau)
$$


with the property for every fixed $\sigma$, the functions $R_{0}^{+} R_{0}^{-} \widetilde{\psi}_{h, n}(\sigma, \cdot)$ and $\left(\widetilde{\psi}_{h, n}-R_{0}^{+} R_{0}^{-} \widetilde{\psi}_{h, n}\right)(\sigma, \cdot)$ are orthogonal in $L^{2}\left(\left(0, h^{-\rho}\right)\right)$. Actually, it follows simply from the definition of $R_{0}^{+}$and $R_{0}^{-}$ that,

$$
\forall \sigma \in \mathbb{R}, \quad \int_{0}^{h^{-\rho}} R_{0}^{+} R_{0}^{-} \widetilde{\psi}_{h, n}(\sigma, \tau) \times\left(\widetilde{\psi}_{h, n}-R_{0}^{+} R_{0}^{-} \widetilde{\psi}_{h, n}\right)(\sigma, \tau) d \tau=0 .
$$

Furthermore, for every fixed $\sigma$, these functions are in the domain of the operator $\mathcal{H}_{0, h}$ in (4.3)(4.4), $R_{0}^{+} R_{0}^{-} \widetilde{\psi}_{h, n}(\sigma, \cdot)$ is in the first eigenspace of the operator $\mathcal{H}_{0, h}$ and $\left(\widetilde{\psi}_{h, n}-R_{0}^{+} R_{0}^{-} \widetilde{\psi}_{h, n}\right)(\sigma, \cdot)$ is in its orthogonal complement. In that way, we get in light of the min-max principle and Lemma 4.1, that a.e. in $\sigma$,

$$
\begin{aligned}
& \int_{0}^{h^{-\rho}}\left|\partial_{\tau} \widetilde{\psi}_{h, n}(\sigma, \tau)\right|^{2} d \tau-\left|\widetilde{\psi}_{h, n}(\sigma, \tau=0)\right|^{2} \\
& \quad \geq \lambda_{1}\left(\mathcal{H}_{0, h}\right) \int_{0}^{h^{-\rho}}\left|R_{0}^{+} R_{0}^{-} \widetilde{\psi}_{h, n}\right|^{2} d \tau+\lambda_{2}\left(\mathcal{H}_{0, h}\right) \int_{0}^{h^{-\rho}}\left|\widetilde{\psi}_{h, n}-R_{0}^{+} R_{0}^{-} \widetilde{\psi}_{h, n}\right|^{2} d \tau \\
& \quad \geq(-1+o(1)) \int_{0}^{h^{-\rho}}\left|R_{0}^{+} R_{0}^{-} \widetilde{\psi}_{h, n}\right|^{2} d \tau
\end{aligned}
$$

Integrating over $\sigma$ in $\left(-h^{-\eta}, h^{-\eta}\right)$ and using also (8.10) and the property that $R_{0}^{+} R_{0}^{-}$is an orthogonal projector, we get

$$
\begin{aligned}
(-h+h o(1)) h & \int_{-h^{-\eta}}^{h^{-\eta}} \int_{0}^{h^{-\rho}}\left|R_{0}^{+} R_{0}^{-} \widetilde{\psi}_{h, n}\right|^{2} d \tau d \sigma \\
\leq & \left(\mu_{n}(h)+\mathcal{O}\left(h^{\frac{3}{2}-\rho}\right)\right) \int_{-h^{-\eta}}^{h^{-\eta}} \int_{0}^{h^{-\rho}}\left|R_{0}^{+} R_{0}^{-} \widetilde{\psi}_{h, n}\right|^{2} d \tau d \sigma \\
& \quad+\left(\mu_{n}(h)+\mathcal{O}\left(h^{\frac{3}{2}-\rho}\right)\right) \int_{-h^{-\eta}}^{h^{-\eta}} \int_{0}^{h^{-\rho}}\left|\widetilde{\psi}_{h, n}-R_{0}^{+} R_{0}^{-} \widetilde{\psi}_{h, n}\right|^{2} d \tau d \sigma .
\end{aligned}
$$

Using that $\mu_{n}(h) \leq-h+h o(1)$ and that $\rho \in\left(0, \frac{1}{2}\right)$, we get

$$
\int_{-h^{-\eta}}^{h^{-\eta}} \int_{0}^{h^{-\rho}}\left|\widetilde{\psi}_{h, n}-R_{0}^{+} R_{0}^{-} \widetilde{\psi}_{h, n}\right|^{2} d \tau d \sigma=o(1) \int_{-h^{-\eta}}^{h^{-\eta}} \int_{0}^{h^{-\rho}}\left|R_{0}^{+} R_{0}^{-} \widetilde{\psi}_{h, n}\right|^{2} d \sigma .
$$

Thus we get (3).

Proof of (4):

From the assertions (2) and (3) and the normalization of $v_{h, n}$, we get

$$
\left\|R_{0}^{+} R_{0}^{-} \widetilde{\psi}_{h, n}\right\|_{2}=1+o(1) .
$$

Using the definition of $R_{0}^{+}$, we get

$$
\left\|R_{0}^{+} R_{0}^{-} \widetilde{\psi}_{h, n}\right\|_{2}=\left\|R_{0}^{-} \widetilde{\psi}_{h, n}\right\|_{2}
$$

and the assertion in (4) follows.

Proof of (5):

The functions $v_{h, i}$ and $v_{h, j}$ are orthogonal if $i \neq j$ in $L^{2}(\Omega)$.

By assertions (2) and (3), we get that $R_{0}^{+} R_{0}^{-} \widetilde{\psi}_{h, i}$ and $R_{0}^{+} R_{0}^{-} \widetilde{\psi}_{h, j}$ are almost orthogonal, i.e. their inner product is $o(1)$. By definition of $R_{0}^{+}$, we have

$$
\left\langle R_{0}^{+} R_{0}^{-} \widetilde{\psi}_{h, i}, R_{0}^{+} R_{0}^{-} \widetilde{\psi}_{h, j}\right\rangle_{L^{2}\left(\mathbb{R}^{2}\right)}=\left\langle R_{0}^{-} \widetilde{\psi}_{h, i}, R_{0}^{-} \widetilde{\psi}_{h, j}\right\rangle_{L^{2}(\mathbb{R})},
$$

and the assertion in (5) follows.

It results from (8.6) the following simple corollary of Proposition 8.1

Corollary 8.2. There holds, 
(1) $\left\|\widetilde{R}_{0, h}^{-} \widetilde{\psi}_{h, n}\right\|_{L^{2}(\mathbb{R})}=1+o(1)$;

(2) If $i \neq j$, then the functions $\widetilde{R}_{0, h}^{-} \widetilde{\psi}_{h, i}$ and $\widetilde{R}_{0, h}^{-} \widetilde{\psi}_{h, j}$ are almost orthogonal in the following sense,

$$
\left\langle\widetilde{R}_{0, h}^{-} \widetilde{\psi}_{h, i}, \widetilde{R}_{0, h}^{-} \widetilde{\psi}_{h, j}\right\rangle_{L^{2}(\mathbb{R})}=o(1) .
$$

8.2.2. Useful identities. Recall the definition of the operators $P_{0}, P_{2}, P_{3}$ and $Q_{h}$ in (6.15), together with the definition of the functions $q_{1}, q_{2}$ and $q_{3}$ in (6.11)-

We introduce the operator

$$
\widetilde{\mathcal{L}}_{h}=-h^{3 / 4} \tilde{a}^{-1} \partial_{\sigma}\left(\tilde{a}^{-1} \partial_{\sigma}\right)-\tilde{a}^{-1} \partial_{\tau}\left(\tilde{a} \partial_{\tau}\right),
$$

with $\widetilde{a}$ as in (6.4).

Lemma 8.3. There holds:

(1) $-\partial_{\tau}^{2} u_{0, h}=-u_{0, h}+\epsilon_{1}(h) u_{0, h}$ and $\partial_{\tau} u_{0, h}=-u_{0, h}+\epsilon_{1}(h) u_{0, h}+\epsilon_{2}(\tau ; h)$ where

$$
\left|\epsilon_{1}(h)\right| \leq C \exp \left(-2 h^{-\rho}\right), \quad\left|\epsilon_{2}(\tau ; h)\right| \leq C \exp \left(-h^{-\rho}\right),
$$

and $C>0$ is a constant.

(2) For all $n \in \mathbb{N}$,

$$
R_{0, h}^{-} \widetilde{\mathcal{L}}_{h} \widetilde{\psi}_{h, n}=\left(-1-h^{1 / 2} \kappa_{\max }\right) R_{0, h}^{-} \widetilde{\psi}_{h, n}+h^{3 / 4} H_{\text {harm }} \widetilde{R}_{0, h}^{-} \widetilde{\psi}_{h, n}+h^{3 / 4} r\left(\widetilde{\psi}_{h, n}\right),
$$

where $k_{2}=-\kappa^{\prime \prime}(0)>0$,

$$
H_{\text {harm }}=-\frac{d^{2}}{d \sigma^{2}}+\frac{k_{2}}{2} \sigma^{2},
$$

and $r\left(\widetilde{\psi}_{h, n}\right)$ is a function of $\sigma$ satisfying for some constant $C>0$ and all sufficiently small $h$,

$$
\left\|r\left(\widetilde{\psi}_{h, n}\right)\right\|_{L^{2}(\mathbb{R})} \leq C h^{\frac{1}{8}-3 \eta} .
$$

Proof. The assertion in (1) follows from (4.9), the definition of $u_{0, h}$ in (4.10) and a straightforward computation.

The functions $u_{0, h}$ and $\widetilde{\psi}_{h, n}$ vanish at $\tau=h^{-\rho}$ and they satisfy the following Robin condition at $\tau=0$,

$$
\partial_{\tau} u_{0, h}=-u_{0, h} \quad \text { and } \quad \partial_{\tau} \widetilde{\psi}_{h, n}=-\psi_{h, n} .
$$

Thus, an integration by parts gives, for a.e. $\sigma$,

$$
\int_{0}^{h^{-\rho}} u_{0, h}(\tau) \widetilde{a}^{-1} \partial_{\tau}\left(\widetilde{a} \partial_{\tau} \widetilde{\psi}_{h, n}\right) \widetilde{a} d \tau=\int_{0}^{h^{-\rho}} \widetilde{a}^{-1} \partial_{\tau}\left(\widetilde{a} \partial_{\tau} u_{0, h}\right) \widetilde{\psi}_{h, n} \widetilde{a} d \tau
$$

The integral in the left hand side can be transformed to a simpler one by using the (point-wise) expression of the operator $a^{-1} \partial_{\tau}\left(\widetilde{a} \partial_{\tau}\right)$ in (6.11). This allows us to write

$$
\begin{aligned}
& -\int_{0}^{h^{-\rho}} u_{0, h}(\tau) \widetilde{a}^{-1} \partial_{\tau}\left(\widetilde{a} \partial_{\tau} \widetilde{\psi}_{h, n}\right) \widetilde{a} d \tau \\
& \quad=\int_{0}^{h^{-\rho}}\left\{\left(P_{0}+h^{1 / 2} P_{2}+h^{3 / 4} \frac{\kappa^{\prime \prime}(0)}{2 !} \sigma^{2} \partial_{\tau}+h^{7 / 8} q_{1}(\sigma, \tau) \partial_{\tau}\right) u_{0, h}\right\} \widetilde{\psi}_{h, n} \widetilde{a} d \tau .
\end{aligned}
$$

The definition of the operators $P_{0}, P_{2}$ in (6.16) and the assertion in (1) give us

$$
\left(P_{0}+h^{1 / 2} P_{2}+h^{3 / 4} \frac{\kappa^{\prime \prime}(0)}{2 !} \sigma^{2} \partial_{\tau}\right) u_{0, h}=\left(-1-\kappa_{\max } h^{1 / 2}+h^{3 / 4} \frac{k_{2}}{2} \sigma^{2}\right) u_{0, h}+e_{h},
$$

where $k_{2}=-\kappa^{\prime \prime}(0)>0$ and

$$
e_{h}(\tau)=\left[1+h^{1 / 2} \kappa_{\max }+h^{3 / 4} \frac{k_{2} \sigma^{2}}{2}\right] \epsilon_{1}(h) u_{0, h}(\tau)+\left[h^{1 / 2} \kappa_{\max }+h^{3 / 4} \frac{k_{2} \sigma^{2}}{2}\right] \epsilon_{2}(\tau ; h) .
$$


Since the variable $\tau$ lives in $\left(0, h^{-\rho}\right), 0<\rho<\frac{1}{2}$ and $\kappa(\cdot)$ is bounded, we get that $\widetilde{a}=1+\mathcal{O}\left(h^{\frac{1}{2}-\rho}\right)$. Now, the properties of the expressions $\epsilon_{1}$ and $\epsilon_{2}$ in assertion (1) yield

$$
\left|\int_{0}^{h^{-\rho}} e_{n}(\tau) \widetilde{\psi}_{h, n}(\sigma, \tau) \widetilde{a} d \tau\right| \leq \mathcal{O}\left(h^{\infty}\right)\left(\int_{0}^{h^{-\rho}}\left|\widetilde{\psi}_{h, n}(\sigma, \tau)\right|^{2} \widetilde{a} d \tau\right)^{1 / 2} .
$$

Using the normalization of $\widetilde{\psi}_{h, n}$ in the $L^{2}$-norm relative to the measure $\widetilde{a} d \sigma d \tau$, we get further,

$$
\int_{\mathbb{R}}\left|\int_{0}^{h^{-\rho}} e_{n}(\tau) \widetilde{\psi}_{h, n}(\sigma, \tau) \widetilde{a} d \tau\right|^{2} d \sigma \leq \mathcal{O}\left(h^{\infty}\right) \int_{\mathbb{R}} \int_{0}^{h^{-\rho}}\left|\widetilde{\psi}_{h, n}(\sigma, \tau)\right|^{2} \widetilde{a} d \tau=\mathcal{O}\left(h^{\infty}\right) .
$$

We infer from (8.12) and (8.13) the following identity

$$
-\int_{0}^{h^{-\rho}} u_{0, h}(\tau) \widetilde{a}^{-1} \partial_{\tau}\left(\widetilde{a} \partial_{\tau} \widetilde{\psi}_{h, n}\right) \widetilde{a} d \tau=\left(-1-h^{1 / 2} \kappa_{\max }+h^{3 / 4} \frac{k_{2}}{2} \sigma^{2}\right) R_{0, h}^{-} \widetilde{\psi}_{h, n}+r_{1}\left(\widetilde{\psi}_{h, n}\right),
$$

where $R_{0, h}^{-} \widetilde{\psi}_{h, n}$ is defined as in (8.4), and the remainder term is a function of the variable $\sigma$ and defined as follows:

$$
r_{1}\left(\widetilde{\psi}_{h, n}\right)=\int_{0}^{h^{-\rho}} e_{h}(\tau) \widetilde{\psi}_{h, n}(\sigma, \tau) \widetilde{a} d \tau+\int_{0}^{h^{-\rho}}\left(h^{7 / 8} q_{1}(\sigma, \tau) \partial_{\tau} u_{0, h}(\tau)\right) \widetilde{\psi}_{h, n}(\sigma, \tau) \widetilde{a} d \tau .
$$

In the support of $\widetilde{\psi}_{h, n},|\sigma| \leq h^{-\eta}$ and $0 \leq \tau \leq h^{-\rho}$. The choice of $\rho$ and $\eta$ is such that $\rho=\frac{1}{8}+\eta$ and $0<\eta<\frac{1}{8}$. Thanks to (6.13),$\left|q_{1}(\sigma, \tau)\right| \leq C\left(|\sigma|^{3}+h^{1 / 8} \tau\right)=\mathcal{O}\left(h^{-3 \eta}\right)$ in the support of $\widetilde{\psi}_{h, n}$. By the Cauchy-Schwarz inequality and the assertion in (1), we may write

$$
\left|\int_{0}^{h^{-\rho}}\left(h^{7 / 8} q_{1}(\sigma, \tau) \partial_{\tau} u_{0, h}(\tau)\right) \widetilde{\psi}_{h, n}(\sigma, \tau) \widetilde{a} d \tau\right| \leq C h^{\frac{7}{8}-3 \eta}\left(\int_{0}^{h^{-\rho}}\left|\widetilde{\psi}_{h, n}(\sigma, \tau)\right|^{2} \widetilde{a}^{2} d \tau\right)^{1 / 2} .
$$

The normalization of $\widetilde{\psi}_{h, n}$ and the estimate $\widetilde{a}=1+\mathcal{O}\left(h^{\frac{1}{2}-\rho}\right)$ yield

$$
\int_{\mathbb{R}}\left|\int_{0}^{h^{-\rho}}\left(h^{7 / 8} q_{1}(\sigma, \tau) \partial_{\tau} u_{0, h}(\tau)\right) \widetilde{\psi}_{h, n}(\sigma, \tau) \widetilde{a} d \tau\right|^{2} d \sigma=\mathcal{O}\left(h^{\frac{7}{8}-3 \eta}\right) .
$$

Inserting this estimate and the one in (8.16) into (8.18), we get that the $L^{2}$-norm in $\mathbb{R}_{\sigma}$ of the remainder term is estimated as follows:

$$
\left\|r_{1}\left(\widetilde{\psi}_{h, n}\right)\right\|_{L^{2}(\mathbb{R})} \leq C h^{\frac{7}{8}-3 \eta} .
$$

Next we look at the term

$$
\begin{aligned}
\int_{0}^{h^{-\rho}} u_{0, h}(\tau) \widetilde{a}^{-1} \partial_{\sigma}\left(\widetilde{a}^{-1} \partial_{\sigma} \widetilde{\psi}_{h, n}\right) \widetilde{a} d \tau=\int_{0}^{h^{-\rho}} u_{0, h}(\tau) \widetilde{a}^{-1} \partial_{\sigma}^{2} \widetilde{\psi}_{h, n} d \tau \\
\quad+\int_{0}^{h^{-\rho}} u_{0, h}(\tau)\left(\widetilde{a}^{-1} \partial_{\sigma} \widetilde{a}^{-1}\right) \partial_{\sigma} \widetilde{\psi}_{h, n} d \tau .
\end{aligned}
$$

Here we will need the operator $\widetilde{R}_{0, h}^{-}$in (8.5). Since the function $u_{0, h}$ is independent of the variable $\sigma$,

Using the simple identity

$$
\int_{0}^{h^{-\rho}} u_{0, h}(\tau) \partial_{\sigma}^{2}\left(\widetilde{a}^{-1} \widetilde{\psi}_{h, n}\right) d \tau=\partial_{\sigma}^{2} \widetilde{R}_{0, h}^{-} \widetilde{\psi}_{h, n}
$$

$$
\widetilde{a}^{-1} \partial_{\sigma}^{2} \widetilde{\psi}_{h, n}=\partial_{\sigma}^{2}\left(\widetilde{a}^{-1} \widetilde{\psi}_{h, n}\right)-2 \partial_{\sigma} \widetilde{a}^{-1} \partial_{\sigma} \widetilde{\psi}_{h, n}-\left(\partial_{\sigma}^{2} \widetilde{a}^{-1}\right) \widetilde{\psi}_{h, n},
$$

we infer from (8.20),

$$
-h^{3 / 4} \int_{0}^{h^{-\rho}} u_{0, h} \widetilde{a}^{-1} \partial_{\sigma}\left(\widetilde{a}^{-1} \partial_{\sigma} \widetilde{\psi}_{h, n}\right) \widetilde{a} d \tau=-h^{3 / 4} \partial_{\sigma}^{2} \widetilde{R}_{0, h}^{-} \widetilde{\psi}_{h, n}+h^{3 / 4} r_{2}\left(\widetilde{\psi}_{h, n}\right),
$$


where the remainder term is a function of the variable $\sigma$ and defined as follows:

$$
r_{2}\left(\widetilde{\psi}_{h, n}\right)=\int_{0}^{h^{-\rho}} u_{0, h}\left[2\left(\partial_{\sigma} \widetilde{a}^{-1}\right) \times\left(\partial_{\sigma} \widetilde{\psi}_{h, n}\right)+\left(\partial_{\sigma}^{2} \widetilde{a}^{-1}\right) \widetilde{\psi}_{h, n}\right] d \tau .
$$

The definition of $\widetilde{a}$ and the boundedness of the curvature yield that, in the support of $\psi_{h, n}$,

$$
\left|\partial_{\sigma} \widetilde{a}^{-1}\right|=\mathcal{O}\left(h^{\frac{1}{2}-\rho} h^{\frac{1}{8}}\right) \quad \text { and } \quad\left|\partial_{\sigma}^{2} \widetilde{a}^{-1}\right|=\mathcal{O}\left(h^{\frac{1}{2}-\rho} h^{\frac{1}{4}}\right) .
$$

Applying the Cauchy-Schwarz ineqaulity, using the normalization of $u_{0, h}$ and the expansion $\widetilde{a}=1+\mathcal{O}\left(h^{\frac{1}{2}-\rho}\right)$, we get

$$
\left\|r_{2}\left(\widetilde{\psi}_{h, n}\right)\right\|_{L^{2}(\mathbb{R})} \leq C h^{\frac{1}{2}-\rho} h^{\frac{1}{8}}\left\|\partial_{\sigma} \widetilde{\psi}_{h, n}\right\|_{2}+C h^{\frac{1}{2}-\rho} h^{1 / 4}\left\|\widetilde{\psi}_{h, n}\right\|_{2} .
$$

Performing the change of variables $(s, t)=\left(h^{1 / 8} \sigma, h^{1 / 2} \tau\right)$, we get (see (8.8)),

$$
\left\|\partial_{\sigma} \widetilde{\psi}_{h, n}\right\|_{2}=h^{\frac{1}{8}}\left\|\partial_{s} \psi_{h, n}\right\|_{2} \quad \text { and } \quad\left\|\widetilde{\psi}_{h, n}\right\|_{2}=\left\|\psi_{h, n}\right\|_{2}=1+o(1) .
$$

Thus, the remainder term satisfies

$$
\left\|r_{2}\left(\widetilde{\psi}_{h, n}\right)\right\|_{L^{2}(\mathbb{R})} \leq C h^{\frac{1}{2}-\rho} h^{1 / 4}\left\|\partial_{s} \psi_{h, n}\right\|_{2}+C h^{\frac{1}{2}-\rho} h^{1 / 4}
$$

We estimate the norm of $\partial_{s} \psi_{h, n}$ as in Remark 5.3. In that way we get

$$
\left\|r_{2}\left(\widetilde{\psi}_{h, n}\right)\right\|_{L^{2}(\mathbb{R})} \leq \widehat{C} h^{\frac{5}{8}-\rho} .
$$

Also, by (8.6) and the definition of $\rho=\frac{1}{8}+\eta$, we have

$$
\left\|\left(R_{0, h}^{-}-\widetilde{R}_{0, h}^{-}\right) \widetilde{\psi}_{h, n}\right\|_{2} \leq C h^{\frac{1}{2}-\rho}=C h^{\frac{3}{8}-\eta} .
$$

We set $r\left(\widetilde{\psi}_{h, n}\right)=h^{-3 / 4} r_{1}\left(\widetilde{\psi}_{h, n}\right)+r_{2}\left(\widetilde{\psi}_{h, n}\right)+\frac{k_{2}}{2} \sigma^{2}\left(R_{0, h}^{-}-\widetilde{R}_{0, h}^{-}\right) \widetilde{\psi}_{h, n}$. Thanks to (8.19), (8.23), (8.24), and the condition $0<\eta<\frac{1}{8}$, we have

$$
\left\|r\left(\widetilde{\psi}_{h, n}\right)\right\|_{L^{2}(\mathbb{R})}=\mathcal{O}\left(h^{\frac{1}{8}-3 \eta}\right) .
$$

On the other hand, we insert (8.21) into (8.20) then collect the obtained identity and (8.17). By virtue of the definition of $\widetilde{\mathcal{L}}_{h}$ in (8.11), we get the identity in Assertion (2).

8.2.3. Validating the approximation. In the next lemma, we show that the functions $\widetilde{R}_{0, h}^{-} \widetilde{\psi}_{h, n}$ yield approximate eigenfunctions for the harmonic oscillator

$$
H_{\text {harm }}=-\frac{d^{2}}{d \sigma^{2}}+\frac{k_{2}}{2} \sigma^{2} .
$$

Lemma 8.4. Let $n \in \mathbb{N}^{*}, \Lambda_{n}=\left(\mu_{n}(h)+h+h^{3 / 2} \kappa_{\max }\right) h^{-7 / 4}$ and $0<\eta<\frac{1}{24}$. There holds,

$$
\left\|\left(H_{\text {harm }}-\Lambda_{n}\right) \widetilde{R}_{0, h}^{-} \widetilde{\psi}_{h, n}\right\|_{2} \leq C\left(h^{\frac{1}{8}-3 \eta}+h^{\frac{3}{8}-\eta}\left|\Lambda_{n}\right|\right)\left\|\widetilde{R}_{0, h}^{-} \widetilde{\psi}_{h, n}\right\|_{2} .
$$

Thanks to Remark 5.4 and Theorem 6.1, we have $\Lambda_{n}=\mathcal{O}(1)$, as $h \rightarrow 0_{+}$.

Proof of Lemma 8.4. We perform the change of variables $(s, t)=\left(h^{1 / 8} \sigma, h^{1 / 2} \tau\right)$ and use (8.8) to write (also we use (6.3) to express the operator $\mathcal{L}_{h}$ in the boundary coordinates),

$$
\widetilde{\mathcal{L}}_{h} \widetilde{\psi}_{h, n}(\sigma, \tau)=h^{5 / 16} h^{-1} \mathcal{L}_{h} \psi_{h, n}(s, t) \quad \text { and } \quad \widetilde{\psi}_{h, n}(\sigma, \tau)=h^{5 / 16} \psi_{h, n}(s, t) .
$$

By Lemma 8.1, we have

$$
\left\|\widetilde{\mathcal{L}}_{h} \widetilde{\psi}_{h, n}-h^{-1} \mu_{n}(h) \widetilde{\psi}_{h, n}\right\|_{2}=\mathcal{O}\left(h^{\infty}\right) .
$$

Since the norm of the operator $R_{0, h}^{-}$in (8.4) is equal to 1 , we have

$$
\left\|R_{0, h}^{-}\left(\widetilde{\mathcal{L}}_{h} \widetilde{\psi}_{h, n}-h^{-1} \mu_{n}(h) \widetilde{\psi}_{h, n}\right)\right\|_{2} \leq\left\|\widetilde{\mathcal{L}}_{h} \widetilde{\psi}_{h, n}-h^{-1} \mu_{n}(h) \widetilde{\psi}_{h, n}\right\|_{2}=\mathcal{O}\left(h^{\infty}\right) .
$$


Now, Lemma 8.3 and (8.6) tell us that

$$
\begin{aligned}
\left\|H_{\text {harm }} \widetilde{R}_{0, h}^{-} \widetilde{\psi}_{h, n}-\Lambda_{n} \widetilde{R}_{0, h}^{-} \widetilde{\psi}_{h, n}\right\|_{L^{2}(\mathbb{R})} & \leq C h^{\frac{1}{8}-3 \eta}+C\left|\Lambda_{n}\right|\left\|R_{0, h}^{-} \widetilde{\psi}_{h, n}-\widetilde{R}_{0, h}^{-} \widetilde{\psi}_{h, n}\right\|_{L^{2}(\mathbb{R})} \\
& \leq C h^{\frac{1}{8}-3 \eta}+C\left|\Lambda_{n}\right| h^{\frac{1}{2}-\rho}=C h^{\frac{1}{8}-3 \eta}+C\left|\Lambda_{n}\right| h^{\frac{3}{8}-\eta} .
\end{aligned}
$$

Corollary 8.2 tells us that the norm of $\widetilde{R}_{0}^{-} \psi_{h, n}$ is $1+o(1)$. In that way, (8.25) finishes the proof of Lemma 8.4.

Lower bounds and three-term asymptotics.

Let $n \in \mathbb{N}^{*}$ be a fixed natural number. Let $W_{n}$ be the space spanned by the functions $\left\{R_{0}^{-} \widetilde{\psi}_{h, j}\right\}_{1 \leq j \leq n}$. These functions are almost orthonormal, see Lemma 8.1. Thanks to Lemma 8.4 and the assumption $0<\eta<\frac{1}{8}$, we see that

$$
\forall \phi \in W_{n}, \quad\left\langle H_{\text {Harm }} \phi, \phi\right\rangle_{L^{2}(\mathbb{R})} \leq\left\{h^{-7 / 4}\left(\mu_{n}(h)+h+h^{3 / 2} \kappa_{\max }\right)(1+o(1))+o(1)\right\}\|\phi\|_{2},
$$

and the dimension of $W_{n}$ is equal to $n$.

By the min-max principle, we deduce that the $n$-th eigenvalue of the harmonic oscillator $H_{\text {harm }}$ satisfies:

$$
(2 n-1) \sqrt{\frac{k_{2}}{2}} \leq h^{-7 / 4}\left(\mu_{n}(h)+h+h^{3 / 2} \kappa_{\max }\right)(1+o(1))+o(1) .
$$

This gives the following lower bound of $\mu_{n}(h)$,

$$
\mu_{n}(h) \geq-h-h^{3 / 2} \kappa_{\max }+(2 n-1) \sqrt{\frac{k_{2}}{2}} h^{7 / 4}+h^{7 / 4} o(1) .
$$

Now, thanks to the upper bound in Theorem 6.1, we get

$$
\mu_{n}(h)=-h-h^{3 / 2} \kappa_{\max }+(2 n-1) \sqrt{\frac{k_{2}}{2}} h^{7 / 4}+h^{7 / 4} o(1) .
$$

Proof of Theorem 2.1.

Let $n \in \mathbb{N}$ and $M \in \mathbb{N} \cup\{0\}$. Thanks to Theorem 7.1, we know that there exists an eigenvalue $\widetilde{\mu}_{n}(h)$ of the operator $\mathcal{L}_{h}$ satisfying as $h \rightarrow 0_{+}$,

$$
\widetilde{\mu}_{n}(h)=-h-h^{3 / 2} \kappa_{\max }+(2 n-1) \sqrt{\frac{k_{2}}{2}} h^{7 / 4}+h^{7 / 4} \sum_{j=0}^{M} \zeta_{j, n} h^{j / 8}+\mathcal{O}\left(h^{\frac{7}{8}+\frac{M+1}{8}}\right) .
$$

Thanks to (8.27), we observe that there exists $h_{0}>0$ such that, for all $h \in\left(0, h_{0}\right)$,

$$
\widetilde{\mu}_{n}(h)=\mu_{n}(h) \text {. }
$$

Thus, $\mu_{n}(h)$ satisfies the asymptotic expansion in Theorem 2.1 .

\section{WKB CONSTRUCTIONS}

In this section, we shortly address the WKB construction in the spirit of Bonnaillie-HérauRaymond [1] (see previously in the context of the Born-Oppenheimer approximation [12]).

We start by recalling the expression in (8.11) of the operator $L_{h}$ in the boundary coordinates $(s, t)$. We will express this operator in the re-scaled coordinates $(s, \tau)=\left(s, h^{-1 / 2} t\right)$. For this purpose it is convenient to introduce the operator

$$
\widehat{L}_{h}=-\partial_{\tau}^{2}-h \hat{a}^{-2} \partial_{s}^{2}-\hat{a}^{-1}\left(\partial_{\tau} \hat{a}\right) \partial_{\tau}-h \hat{a}^{-1}\left(\partial_{s} \hat{a}^{-1}\right) \partial_{s},
$$

where

$$
\hat{a}(s, \tau)=1-h^{1 / 2} \tau \kappa(s), \quad \partial_{\tau} \hat{a}(s, \tau)=-h^{1 / 2} \kappa(s), \quad \partial_{s} \hat{a}^{-1}(s, \tau)=h^{1 / 2} \hat{a}^{-2}(s, \tau) \kappa^{\prime}(s) .
$$

In the $(s, \tau)$ coordinates, the operator $\mathcal{L}_{h}$ is expressed as follows,

$$
\mathcal{L}_{h}=h \widehat{L}_{h} \text {. }
$$


We will abuse notation and identify $\hat{a}^{-1}, \hat{a}^{-2}$ and $\hat{a}^{-3}$ with the series expansions in $\tau$,

$$
\begin{aligned}
\hat{a}^{-1}(s, \tau)=\sum_{j=0}^{\infty} h^{j / 2} \tau^{j}(\kappa(s))^{j}, \quad \hat{a}^{-2}(s, \tau)=\sum_{j=0}^{\infty} c_{j} h^{j / 2} \tau^{j}(\kappa(s))^{j}, & \\
\hat{a}^{-3}(s, \tau) & =\sum_{j=0}^{\infty} d_{j} h^{j / 2} \tau^{j}(\kappa(s))^{j},
\end{aligned}
$$

where, for all $j \in\{0,1, \cdots\}$,

$$
c_{0}=d_{0}=1, \quad c_{j}=\frac{1}{j !} \prod_{k=0}^{j-1}(k-2), \quad d_{j}=\frac{1}{j !} \prod_{k=0}^{j-1}(k-3) \quad(j \geq 1) .
$$

This leads to the following expansion of the operator $\widehat{L}_{h}$,

$$
\begin{aligned}
\widehat{L}_{h} & =-\partial_{\tau}^{2}-h \partial_{s}^{2} \\
& +2 h^{3 / 2} \tau \kappa(s) \partial_{s}^{2}+h^{1 / 2} \partial_{\tau}+h^{3 / 2} \tau \kappa^{\prime}(s) \partial_{s} \\
& -\sum_{j=1}^{\infty} c_{j} h^{\frac{j+2}{2}} \tau^{j}(\kappa(s))^{j} \partial_{s}^{2}+\sum_{j=1}^{\infty} h^{\frac{j+1}{2}} \tau^{j}(\kappa(s))^{j+1} \partial_{\tau}-\kappa^{\prime}(s) \sum_{j=1}^{\infty} h^{\frac{j+3}{2}} d_{j} \tau^{j}(\kappa(s))^{j} \partial_{s} .
\end{aligned}
$$

We use the idea à la Born-Oppenheimer [2, 12, 14] and look for a trial state $\Psi^{W K B}(s, \tau, h)$ having the WKB expansion]

$$
\Psi^{W K B}(s, \tau, h) \sim \exp \left(-\frac{\vartheta(s)}{h^{\frac{1}{4}}}\right)\left(\sum_{\ell=0}^{\infty} a_{\ell}(s, \tau) h^{\frac{\ell}{4}}\right)
$$

and a corresponding eigenvalue

$$
\mu^{W K B} \sim \sum_{\ell=0}^{\infty} \mu_{\ell} h^{\frac{\ell}{4}}
$$

where $\vartheta(s)$ is a real-valued function to be determined.

As we shall see, the analysis below will allow us to select $\mu_{0}, \mu_{1}, \cdots$ so that $\mu^{W K B}$ is an expansion of the ground state energy of the operator $\mathcal{L}_{h}$ (this in particularly means that we impose the Robin condition). The focus on the ground state energy is for the sake of simplicity. A slight modification of the method allows us to get expansions of all the low-lying eigenvalues of the operator $\mathcal{L}_{h}$.

We will arrange the terms in the equation

$$
\widehat{L}_{h} \Psi^{W K B} \sim \mu^{W K B} \Psi^{W K B},
$$

in the form of power series in $h^{1 / 4}$ and select $a_{\ell}(s, \tau)$ and $\mu_{\ell}$ by matching the terms with coefficient $h^{\ell / 4}$.

We introduce the (formal) operator

$$
\widehat{L}_{h}^{\vartheta}:=\exp \left(\frac{\vartheta(s)}{h^{\frac{1}{4}}}\right) \widehat{L}_{h} \exp \left(-\frac{\vartheta(s)}{h^{\frac{1}{4}}}\right),
$$

\footnotetext{
${ }^{1}$ More correctly, this means that

$$
\exp \left(\frac{\vartheta(s)}{h^{\frac{1}{4}}}\right) \Psi^{W K B}(s, \tau, h) \sim \sum_{\ell=0}^{\infty} a_{\ell}(s, \tau) h^{\frac{\ell}{4}} .
$$
}


and notice that, (9.2) is (formally) equivalent to

$$
\widehat{L}_{h}^{\vartheta}\left(\sum_{\ell} a_{\ell}(s, \tau) h^{\frac{\ell}{4}}\right) \sim \mu^{W K B}\left(\sum_{\ell} a_{\ell}(s, \tau) h^{\frac{\ell}{4}}\right) .
$$

The next step is to (formally) expand the operator $\widehat{L}_{h}^{\vartheta}$ as follows:

$$
\widehat{L}_{h}^{\vartheta}=\sum_{\ell=0}^{\infty} Q_{\ell}^{\vartheta} h^{\frac{\ell}{4}} .
$$

In order to get the expressions of the operators $Q_{\ell}^{\vartheta}$, we do a straightforward (but long!) calculation and observe that

$$
\begin{aligned}
\widehat{L}_{h}^{\vartheta}= & -\partial_{\tau}^{2}+h^{\frac{1}{2}}\left(\partial_{\tau}-\vartheta(s)^{2}\right)+h^{\frac{3}{4}}\left(2 \vartheta^{\prime}(s) \partial_{s}+\vartheta^{\prime \prime}(s)\right) \\
& +h^{\frac{4}{4}}\left(-\partial_{s}^{2}+c_{3} \tau^{3} \kappa(s)^{3}+\tau^{3}(\kappa(s))^{5}\right) \\
& +h^{\frac{5}{4}}\left(2 c_{3} \tau^{3} \kappa(s)^{3} \vartheta^{\prime}(s) \partial_{s}-c_{3} \vartheta^{\prime \prime}(s) \kappa(s)^{3}-\tau \kappa^{\prime}(s) \vartheta^{\prime}(s)\right) \\
& +h^{\frac{6}{4}}\left(-c_{5} \tau^{5} \kappa(s)^{5} \partial_{s}^{2}+c_{4} \tau^{4} \vartheta^{\prime}(s) \kappa(s)^{4}+\tau^{4} \kappa(s)^{5}\right) \\
& +\sum_{j=3}^{\infty} h^{\frac{2 j+1}{4}} \tau^{j+1} \kappa(s)^{j+1}\left(2 c_{j+1} \vartheta^{\prime}(s) \partial_{s}-c_{j+1} \vartheta^{\prime \prime}(s)+d_{j+2} \tau \vartheta^{\prime}(s) \kappa^{\prime}(s) \kappa(s)\right) \\
& +\sum_{j=4}^{\infty} h^{\frac{2 j}{4}} \tau^{j+1} \kappa(s)^{j+1}\left(-c_{j+2} \tau \kappa(s) \partial_{s}^{2}-d_{j+3} \tau^{2} \kappa^{\prime}(s) \kappa(s)^{2} \partial_{s}-c_{j+1} \vartheta^{\prime}(s)\right) \\
& +\sum_{j=4}^{\infty} h^{\frac{2 j}{4}} \tau^{j+1} \kappa(s)^{j+2} \partial_{\tau} .
\end{aligned}
$$

In that way, we find the following expressions of the operators in (9.4),

$$
\begin{aligned}
& Q_{0}^{\vartheta}=-\partial_{\tau}^{2}, \\
& Q_{1}^{\vartheta}=0, \\
& Q_{2}^{\vartheta}=\kappa(s) \partial_{\tau}-\vartheta^{\prime}(s)^{2}, \\
& Q_{3}^{\vartheta}=2 \vartheta^{\prime}(s) \partial_{s}+\vartheta^{\prime \prime}(s), \\
& Q_{4}^{\vartheta}=-\partial_{s}^{2}+c_{3} \tau^{3} \kappa(s)^{3}+\tau^{3}(\kappa(s))^{4} \partial_{\tau}, \\
& Q_{5}^{\vartheta}=2 c_{3} \tau^{3} \kappa(s)^{3} \vartheta^{\prime}(s) \partial_{s}-c_{3} \vartheta^{\prime \prime}(s) \kappa(s)^{3}-\tau \kappa^{\prime}(s) \vartheta^{\prime}(s), \\
& Q_{6}^{\vartheta}=-c_{5} \tau^{5} \kappa(s)^{5} \partial_{s}^{2}+c_{4} \tau^{4} \vartheta^{\prime}(s) \kappa(s)^{4}+\tau^{4}(\kappa(s))^{5} \partial_{\tau},
\end{aligned}
$$

and for all $\ell>6$, we have when $\ell$ is even,

$$
Q_{\ell}^{\vartheta}=\tau^{\frac{\ell}{2}+1} \kappa(s)^{\frac{\ell}{2}+1}\left(-c_{\frac{\ell}{2}+2} \tau \kappa(s) \partial_{s}^{2}-d_{\frac{\ell}{2}+3} \tau^{2} \kappa^{\prime}(s) \kappa(s)^{2} \partial_{s}-c_{\frac{\ell}{2}+1} \vartheta^{\prime}(s)+\kappa(s) \partial_{\tau}\right),
$$

and when $\ell$ is odd,

$$
Q_{\ell}^{\vartheta}=\tau^{\frac{\ell-1}{4}+1} \kappa(s)^{\frac{\ell-1}{4}+1}\left(2 c_{\frac{\ell-1}{4}+1} \vartheta^{\prime}(s) \partial_{s}-c_{\frac{\ell-1}{4}+1} \vartheta^{\prime \prime}(s)+d_{\frac{\ell-1}{4}+2} \tau \vartheta^{\prime}(s) \kappa^{\prime}(s) \kappa(s)\right) .
$$

We return to the formal equation in (9.4). The term with coefficient $h^{0}$ yields the equation,

$$
\left(Q_{0}^{\vartheta}-\mu_{0}\right) a_{0}(s, \tau)=0 .
$$

The operator $Q_{0}^{\vartheta}$ is nothing else than the operator $I \otimes \mathcal{H}_{00}$ on $L^{2}\left(\mathbb{R}_{s} \times \mathbb{R}_{+, \tau}\right)$. Hence we will choose $a_{0}$ and later the $a_{j}$ in the domain of $Q_{0}^{\vartheta}$ in order to respect the Robin condition.

This leads us naturally (considering the operator $\mathcal{H}_{0,0}$ introduced in (4.11) to the choice

$$
\mu_{0}=-1 \quad \text { and } \quad a_{0}(s, \tau)=\xi_{0}(s) u_{0}(\tau) .
$$


Since $Q_{1}^{\vartheta}=0$, the term in (9.4) with coefficient $h^{1 / 4}$ yields

$$
\left(Q_{0}^{\vartheta}-\mu_{0}\right) a_{1}(s, \tau)-\mu_{1} a_{1}(s, \tau)=0 .
$$

This leads us to the natural choice $\mu_{1}=0$ and

$$
a_{1}(s, \tau)=\xi_{1}(s) u_{0}(\tau) .
$$

We look at the term with coefficient $h^{1 / 2}$ to obtain

$$
\left(Q_{0}^{\vartheta}-\mu_{0}\right) a_{2}+\left(Q_{2}^{\vartheta}-\mu_{2}\right) a_{0}=0 .
$$

Remembering that $\mu_{0}=-1$ and $a_{0}(s, \tau)=\xi_{0}(s) u_{0}(\tau)$, we get

$$
\left(Q_{0}^{\vartheta}+1\right) a_{2}+u_{0}(\tau)\left(\kappa(s)-\vartheta^{\prime}(s)^{2}-\mu_{2}\right) \xi_{0}(s)=0 .
$$

Multiplying by $u_{0}$ and integrating over $\tau$ leads us to the eikonal equation

$$
-\kappa(s)-\vartheta^{\prime}(s)^{2}-\mu_{2}=0 .
$$

Consequently, we take $\mu_{2}=-\kappa(0)$, impose the condition $\vartheta^{\prime}(0)=0$ and determine $\vartheta$ from (9.5). In particular, we obtain by differentiating (9.5) two successive times,

$$
\vartheta^{\prime \prime}(0)=\sqrt{-\frac{1}{2} \kappa^{\prime \prime}(0)} .
$$

We take $a_{2}$ in the form

$$
a_{2}(s, \tau)=\xi_{2}(s) u_{0}(\tau),
$$

so that the term $\left(Q_{0}^{\vartheta}+1\right) a_{2}$ vanishes.

Now we look at the term with coefficient $h^{3 / 4}$ in (9.4). This yields

$$
\left(Q_{0}^{\vartheta}+1\right) a_{3}+\left(Q_{2}^{\vartheta}-\mu_{2}\right) a_{1}+\left(Q_{3}^{\vartheta}-\mu_{3}\right) a_{0}=0 .
$$

Using (9.5), we see that the term $\left(Q_{2}^{\vartheta}-\mu_{2}\right) a_{1}$ vanishes. Choosing

$$
a_{3}(s, \tau)=\xi_{3}(s) u_{0}(\tau) .
$$

forces the term $\left(Q_{0}^{\vartheta}+1\right) a_{3}$ to vanish as well. We end up with the equation

$$
\left(Q_{3}^{\vartheta}-\mu_{3}\right) a_{0}=0 .
$$

Multiplying by $u_{0}$ and integrating over $\tau$ gives us the first transport equation

$$
2 \vartheta^{\prime}(s) \xi_{0}^{\prime}(s)+\left(\vartheta^{\prime \prime}(s)-\mu_{3}\right) \xi_{0}(s)=0 .
$$

In order to respect the condition $\vartheta^{\prime}(0)=0$, 9.7 leads us to choose

$$
\mu_{3}=\vartheta^{\prime \prime}(0)=\sqrt{-\frac{1}{2} \kappa^{\prime \prime}(0)}
$$

We can determine $\xi_{0}(s) \neq 0$ by solving (9.7) in a neighborhood of $s=0$ and find:

$$
\xi_{0}(s)=c_{0} \exp \left(-\frac{\vartheta^{\prime \prime}(s)-\vartheta^{\prime \prime}(0)}{2 \vartheta^{\prime}(s)}\right)
$$

where $c_{0} \neq 0$ is a constant. 
The iteration process.

In (9.4), the term with coefficient $h^{\ell / 4}, \ell \geq 4$, leads us to the equation,

$$
\sum_{k=0}^{\ell}\left(Q_{k}^{\vartheta}-\mu_{k}\right) a_{\ell-k}=0 .
$$

We need to define $a_{\ell}$ and $\mu_{\ell}$ for all $\ell \geq 4$. We will do this by iteration.

Let us start by examining the case $\ell=4$. Thanks to the conditions in (9.5), (9.8) becomes

$$
\left(Q_{0}^{\vartheta}+1\right) a_{4}+\left(Q_{3}^{\vartheta}-\mu_{3}\right) a_{1}+\left(Q_{4}^{\vartheta}-\mu_{4}\right) a_{0}=0 .
$$

Multiplying by $u_{0}(\tau)$ and integrating over $\tau$ leads us to the equation,

$$
2 \vartheta^{\prime}(s) \xi_{1}^{\prime}(s)+\left(\vartheta^{\prime \prime}(s)-\mu_{3}\right) \xi_{1}(s)+F_{1}(s)-\mu_{4} \xi_{0}(s)=0,
$$

where

We select $\mu_{4}$ such that

$$
F_{1}(s)=\int_{0}^{\infty} u_{0}(\tau) Q_{4}^{\vartheta} a_{0}(s, \tau) d \tau
$$

and impose the condition

$$
F_{1}(0)-\mu_{4} \xi_{0}(0)=0
$$

$$
\xi_{1}(0)=0 \text {. }
$$

Note that this choice is consistent with (9.10), thanks to $\mu_{3}=\vartheta^{\prime \prime}(0)$ and $\vartheta^{\prime}(0)=0$.

The assumption on the curvature is that it attains its maximal value at the unique point $s=0$.

Notice that, by (9.5), for $s \in\left(-\frac{|\partial \Omega|}{2}, \frac{|\partial \Omega|}{2}\right), \vartheta^{\prime}$ does not vanish except at 0 and that

where $k_{2}=-\kappa^{\prime \prime}(0)>0$.

$$
2 \vartheta^{\prime}(s) \sim \sqrt{2 k_{2}} s \text { and } \vartheta^{\prime \prime}(s) \sim \sqrt{\frac{k_{2}}{2}}
$$

Dividing (9.10) by $2 \vartheta^{\prime}$, we get a first order ordinary equation with $C^{\infty}$ coefficients in $\left(-\frac{|\partial \Omega|}{2}, \frac{|\partial \Omega|}{2}\right)$ which admits a unique explicit solution $\xi_{1}(s)$ (by the method of the variation of constants), if we impose the condition $\xi_{1}(0)=0$.

With this condition the function $v_{4}=\left(Q_{3}^{\vartheta}-\mu_{3}\right) a_{1}-\left(Q_{4}^{\vartheta}-\mu_{4}\right) a_{0}$ is, for any $s$, orthogonal to the function $u_{0}$ in $L^{2}\left(\mathbb{R}_{+}\right)$.

We return to (9.9) and define $a_{4}(s, \tau)$ by

$$
a_{4}(s, \tau)=\xi_{4}(s) u_{0}(\tau)+\left(Q_{0}^{\vartheta}+1\right)^{-1} v_{4}(s, \tau),
$$

where $\xi_{4}(s)$ will be selected later in the definition of $\mu_{7}$ and $a_{7}$.

Clearly, $a_{4}(s, \tau)$ is of the form,

$$
a_{4}(s, \tau)=\xi_{4}(s) u_{0}(\tau)+\sum_{k=1}^{n_{4}} \xi_{4, k}(s) u_{4, k}(\tau) .
$$

Now we describe the iteration process. Let $\ell \geq 5$ and $\xi_{k}(s), k \in\{4, \cdots, \ell\}$, be smooth functions. Suppose that, $\left(\xi_{k}(s)\right)_{k=0}^{\ell-3}$ and for all $M \in\{0, \cdots, \ell-1\}, \mu_{M} \in \mathbb{R}$ and

$$
a_{M}=\xi_{M}(s) u_{0}(\tau)+\sum_{k=1}^{n_{M}} \xi_{M, k}(s) u_{M, k}(\tau)
$$

satisfy

We want to select $\mu_{\ell} \in \mathbb{R}$ and

$$
\sum_{k=0}^{M}\left(Q_{k}^{\vartheta}-\mu_{k}\right) a_{M-k}=0 .
$$

$$
a_{\ell}=\xi_{\ell}(s) u_{0}(\tau)+\sum_{k=1}^{n_{\ell}} \xi_{\ell, k}(s) u_{\ell, k}(\tau)
$$


such that (9.8) is satisfied. We expand (9.8) as follows:

$$
\left(Q_{0}^{\vartheta}+1\right) a_{\ell}+\left(Q_{3}^{\vartheta}-\mu_{3}\right) a_{\ell-3}+G_{\ell-3}+\left(Q_{\ell}^{\vartheta}-\mu_{\ell}\right) a_{0}=0
$$

where

$$
G_{\ell-3}(s, \tau)=\sum_{k=4}^{\ell-1}\left(Q_{k}^{\vartheta}-\mu_{k}\right) a_{\ell-k} .
$$

Multiplying by $u_{0}(\tau)$ and integrating over $\tau$ yields

$$
2 \vartheta^{\prime}(s) \xi_{\ell-3}^{\prime}(s)+\left(\vartheta^{\prime \prime}(s)-\mu_{3}\right) \xi_{\ell-3}(s)+F_{\ell-3}(s)-\mu_{\ell} \xi_{0}(s)=0,
$$

where

$$
F_{\ell-3}(s)=\int_{0}^{\infty}\left\{\left(Q_{3}^{\vartheta}-\mu_{3}\right)\left(\sum_{k=1}^{n_{M}} \xi_{\ell-3, k}(s) u_{\ell-3, k}(\tau)\right)+G_{\ell-3}(s, \tau)+Q_{\ell}^{\vartheta} a_{0}(s, \tau)\right\} u_{0}(\tau) d \tau .
$$

We select $\mu_{\ell}$ such that

$$
F_{\ell-3}(0)-\mu_{\ell} \xi_{0}(0)=0
$$

and impose the following condition

$$
\xi_{\ell}(0)=0
$$

We divide both sides of (9.12) by $2 \vartheta^{\prime}(s)$ and get a first order differential equation in $\xi_{\ell-3}(s)$. Under the condition $\xi_{\ell-3}(0)=0$, this equation has a unique solution $\xi_{\ell-3}(s)$ defined in $\left(-\frac{|\partial \Omega|}{2}, \frac{|\partial \Omega|}{2}\right)$. Then we define $a_{\ell}(s, \tau)$ as follows:

$$
a_{\ell}(s, \tau)=\xi_{\ell}(s) u_{0}(\tau)+\left(Q_{0}^{\vartheta}+1\right)^{-1}\left(v_{\ell-3}(\sigma, \tau)\right),
$$

where

$$
v_{\ell-3}(\sigma, \tau)=-\left(Q_{3}^{\vartheta}-\mu_{3}\right) a_{\ell-3}-G_{\ell-3}-\left(Q_{\ell}^{\vartheta}-\mu_{\ell}\right) a_{0} .
$$

\section{REFERENCES}

[1] V. Bonnaillie, F. Hérau, and N. Raymond. Magnetic WKB expansions. HAL : hal-00966003, version 2, arXiv : 1405.7157 (2014).

[2] M. Born, R. Oppenheimer. Zur Quantentheorie der Molekeln. Ann. Phys. 84, 457-484 (1927).

[3] P. Exner, A. Minakov, L. Parnovski. Asymptotic eigenvalue estimates for a Robin problem with a large parameter. Portugal. Math. 71 (2) 141-156 (2014).

[4] S. Fournais, B. Helffer. Spectral Methods in Surface Superconductivity. Progress in Nonlinear Differential Equations and Their Applications, Vol. 77, Birkhäuser (2010).

[5] S. Fournais, B. Helffer. Accurate eigenvalue asymptotics for the magnetic Neumann Laplacian. Ann. Inst. Fourier. 56 (1) 1-67 (2006).

[6] B. Helffer, A. Morame. Magnetic bottles in connection with superconductivity. J. Func. Anal. 181 (2) 604-680 (2001).

[7] B. Helffer, K. Pankrashkin. Tunneling between corners for Robin Laplacians. Preprint 2014 : arXiv:1404.4765. To appear in J. London Math. Soc.

[8] B. Helffer, J. Sjöstrand. Multiple wells in the semiclassical limit I. Comm. Partial Differ. Equations 9 (4) 337-408 (1984) .

[9] A. Kachmar, M. Persson. On the essential spectrum of magnetic Schrödinger operators in exterior domains. Arab J. Math. Sci. 19 (2) 217-222 (2013).

[10] A. Kachmar. On the ground state energy for a magnetic Schrödinger operator and the effect of the de Gennes boundary conditions. C.R. Math. Acad. Sci. Paris 332, 701-706 (2006).

[11] A. Kachmar. On the ground state energy for a magnetic Schrödinger operator and the effect of the de Gennes boundary conditions. J. Math. Phys. 47(7), 072106, 32 pp (2006).

[12] S. Lefebvre. Semiclassical analysis of the operator $h^{2} D_{x}^{2}+D_{y}^{2}+\left(1+x^{2}\right) y^{2}$. Manuscript June 1986. Unpublished.

[13] M. Levitin, L. Parnovski. On the principal eigenvalue of a Robin problem with a large parameter. Math. Nachr. 281, 272-281 (2008).

[14] A. Martinez. Développements asymptotiques et effet tunnel dans l'approximation de Born-Oppenheimer. Ann. Inst. Henri Poincaré, Sect. Phys. Théor. 50 (3), 239-257 (1989).

[15] K. Pankrashkin. On the asymptotics of the principal eigenvalue problem for a Robin problem with a large parameter in a planar domain. Nanosystems: Physics, Chemistry, Mathematics, 20134 (4), 474-483. 
[16] K. Pankrashkin, N. Popoff. Mean curvature bounds and eigenvalues of Robin Laplacians. Preprint July 2014. arXiv:1407.3087

(B. Helffer) Université de Paris-Sud, Bât 425, 91405 Orsay, France and Laboratoire Jean Leray, Université de Nantes, France.

E-mail address: bernard.helffer@math.u-psud.fr

(A. Kachmar) Lebanese University, Department of Mathematics, Hadath, Lebanon.

E-mail address: ayman.kashmar@gmail.com 